\title{
A mega-aggregation framework synthesis of the barriers and facilitators to linkage, adherence to ART and retention in care among people living with HIV
}

Lynn Hendricks ${ }^{1,2^{*}}$ (D) Ingrid Eshun-Wilson ${ }^{1}$ and Anke Rohwer ${ }^{1}$ (D)

\begin{abstract}
Background: People living with human immunodeficiency virus (PLHIV) struggle with the challenges of living with a chronic disease and integrating antiretroviral treatment (ART) and care into their daily lives. The aims of this study were as follows: (1) to undertake the first mega-aggregation of qualitative evidence syntheses using the methods of framework synthesis and (2) make sense of existing qualitative evidence syntheses that explore the barriers and facilitators of adherence to antiretroviral treatment, linkage to care and retention in care for PLHIV to identify research gaps.

Methods: We conducted a comprehensive search and did all screening, data extraction and critical appraisal independently and in duplicate. We used the Kaufman HIV Behaviour Change model (Kaufman et al., 2014) as a framework to synthesise the findings using the mega-aggregative framework synthesis approach, which consists of 8 steps: (1) identify a clearly defined review question and objectives, (2) identify a theoretical framework or model, (3) decide on criteria for considering reviews for inclusion, (4) conduct searching and screening, (5) conduct quality appraisal of the included studies, (6) data extraction and categorisation, (7) present and synthesise the findings, and (8) transparent reporting. We evaluated systematic reviews up to July 2018 and assessed methodological quality, across reviews, using the Joanna Briggs Institute Critical Appraisal Checklist for Systematic Reviews.

(Continued on next page)
\end{abstract}

\footnotetext{
* Correspondence: Iynnah@sun.ac.za

'Centre for Evidence-Based Health Care, Division Epidemiology and Biostatistics, Faculty of Medicine and Health Sciences, Stellenbosch University, Cape Town, South Africa

${ }^{2}$ Social, Methodological, Innovative, Kreative, Centre for Sociological Research,

Faculty of Social Sciences, Katholieke Universiteit Leuven, Leuven, Belgium
}

C C The Author(s). 2021 Open Access This article is licensed under a Creative Commons Attribution 4.0 International License, which permits use, sharing, adaptation, distribution and reproduction in any medium or format, as long as you give appropriate credit to the original author(s) and the source, provide a link to the Creative Commons licence, and indicate if changes were made. The images or other third party material in this article are included in the article's Creative Commons licence, unless indicated otherwise in a credit line to the material. If material is not included in the article's Creative Commons licence and your intended use is not permitted by statutory regulation or exceeds the permitted use, you will need to obtain permission directly from the copyright holder. To view a copy of this licence, visit http://creativecommons.org/licenses/by/4.0/ The Creative Commons Public Domain Dedication waiver (http://creativecommons.org/publicdomain/zero/1.0/) applies to the data made available in this article, unless otherwise stated in a credit line to the data. 
(Continued from previous page)

Results: We included 33 systematic reviews from low, middle- and high-income countries, which reported on 1, 111,964 PLHIV. The methodological quality of included reviews varied considerably. We identified 544 unique thirdorder concepts from the included systematic reviews, which were reclassified into 45 fourth-order themes within the individual, interpersonal, community, institutional and structural levels of the Kaufman HIV Behaviour Change model. We found that the main influencers of linkage, adherence and retention behaviours were psychosocial and personal characteristics - perceptions of ART, desires, fears, experiences of HIV and ART, coping strategies and mental health issues -interwoven with other factors on the interpersonal, community, institutional and structural level. Using this approach, we found interdependence between factors influencing ART linkage, retention and adherence and identified the need for qualitative evidence that explores, in greater depth, the complex relationships between structural factors and adherence, sociodemographic factors such as community violence and retention, and the experiences of growing up with HIV in low- and middle-income countries-specifically in children, youth, women and key populations.

Conclusions: This is the first mega-aggregation framework synthesis, or synthesis of qualitative evidence syntheses using the methods of framework synthesis at the overview level. We found the novel method to be a transparent and efficient method for assessing the quality and making sense of existing qualitative systematic reviews.

Systematic review registration: The protocol of this overview was registered on PROSPERO (CRD42017078155) on 17 December 2017.

Keywords: Overview, Mega-aggregation, Qualitative, Synthesis, Human immunodeficiency virus, Linkage, Adherence, Retention, ART

\section{Background}

Qualitative evidence syntheses (QES) aggregate, integrate and interpret results from primary qualitative studies [1]. Like quantitative systematic reviews, QES follow transparent, systematic and rigorous methods. With the increase in number of QES on HIV adherence research [2], the next step is to provide an overview of existing systematic reviews to identify research gaps and an up-to-date synthesis of what is known. An overview is also referred to by names such as an umbrella review or a review of reviews. Methods of QES are complex and continue to develop [3]. Although there is guidance on summarising qualitative systematic reviews $[4,5]$, the application of the guidance to cases is still emerging in the literature, with examples of meta-summary of reviews [6, 7] and an application of mega-ethnography [8]. To consider evidence with the aim of assessing the quality of the existing evidence, identifying research gaps to formulate new research questions, or to make decisions about best practice, the appropriate QES method would be meta-aggregation. Metaaggregation does not aim to produce deeper interpretative analysis of the data extracted from the primary studies but rather summarises findings to produce recommendations for action $[9,10]$. Introducing an existing theory or framework [11] into this process can contribute to the efficiency, rigour and pragmatism of meta-aggregation. In this study, we illustrate mega-aggregation framework synthesis to make sense of existing qualitative systematic reviews.

Exploring barriers and facilitators of antiretroviral therapy Although access to HIV care has improved significantly over the past few years, people living with HIV (PLHIV) still face numerous challenges when it comes to initiating care and staying on treatment. Human immunodeficiency virus (HIV) represents one of the greatest global public health challenge in history, and since the beginning of the epidemic, approximately 78 million people worldwide have been infected with HIV and 35 million people have died [12]. The Joint United Nations Programme on HIV/ acquired immunodeficiency syndrome (AIDS) (UNAIDS) set the global 90-90-90-target to combat HIV infection by 2020 [13]. The goal aims for $90 \%$ of all people to know their HIV status, of those who test positive, $90 \%$ should be linked to care, and of those being adherent to care, 90\% will have achieved viral suppression. Nearly 37 million people were estimated to be living with HIV worldwide in 2017; however, only 60\% were aware of their HIV status and only $49 \%$ of those who knew their status were accessing treatment [14]. The HIV burden varies considerably between countries, with regions in Africa having the highest HIV prevalence with HIV being the leading cause of death in South Africa [12]. To date there is no known cure for AIDS. However, being linked to care and adhering to antiretroviral therapy (ART) has been shown to improve quality of life for PLHIV, and in most cases provided similar life expectancy periods for those without HIV $[15,16]$.

\section{Why it is important to do this overview}

PLHIV continue to be challenged by the complexities related to being HIV positive and integrating ART treatment and care into their daily lives. Unsuccessful interventions and the target driven 90-90-90 goals have increased researchers' commitment to understanding the 
human experience of living with HIV and engaging in the HIV treatment cascade. Some reviews have focused only on prevention, adherence, linkage to care or retention in care. This can lead to an abundance of research in one area on the cascade and neglect of others. With the growing body of existing systematic reviews [17, 18], there is no coherent sense of what is already known across populations and settings, and there is uncertainty about the quality of the existing evidence. This can make it hard for policy makers and practitioners to make evidence-informed decisions. However, the increase in QES makes research innovation in the synthesis of qualitative review-level evidence possible. The overarching aim of this study is to debut the first application of mega-aggregation framework synthesis to qualitative systematic review-level evidence. This method of summarising QES in an overview will be applied to review-level evidence of barriers and facilitators of linkage to care, adherence to ART and retention in care for PLHIV in low-, middle- and high-income countries.

\section{Methods \\ Paradigmatic stance}

Overviews of reviews aim to provide a single synthesis or summary from multiple systematic reviews [19]. QES often have a theoretical underpinning to understand findings and interpret meaning. Qualitative research is usually positioned in the interpretive or critical-realist paradigm. Another approach to QES is metaaggregation, which is based on the philosophy of pragmatism $[20,21]$ and users of this method aim for immediate usability of the review findings. This study proposes the application of mega-aggregation, which, unlike mega-ethnography, does not focus on the generation of new theory nor aims to provide deepened conceptual interpretations of findings [8], but rather aims to provide an overview of the existing evidence, identify evidence gaps and make recommendations for future research or immediate action [21, 22].

\section{Overview design}

In the context of the pragmatic stance and the anticipated large number of existing systematic reviews, a predetermined theoretical framework [23] with broad categories was selected to guide the aggregation and synthesis within this overview, which built on the steps in methods development for conducting overviews [24], QES [25, 26], systematic review synthesis $[19,27,28]$, meta-aggregation [9, $20,21]$ and framework synthesis $[29,30]$. The novel approach of mega-aggregation framework synthesis was developed and utilised to identify evidence gaps and to inform future research from the evidence collated within included systematic reviews. The mega-aggregative framework synthesis approach consists of eight distinct steps
(Fig. 1). The steps are as follows: (1) identify a clearly defined review question and objectives, (2) identify a theoretical framework or model, (3) decide on criteria for considering reviews for inclusion, (4) conduct searching and screening, (5) conduct quality appraisal of the included studies (although some may prefer not too), (6) data extraction and categorisation, (7) present and synthesise the findings, and (8) transparent reporting.

\section{Step 1: identifying a clearly defined research question and objectives}

This study aimed to answer the question:

What is the available review-level evidence of the selfreported barriers and facilitators to linkage, adherence to ART and retention in care, for people living with HIV in low-, middle- and high-income countries?

The primary objective was to gather, appraise and synthesise the systematic review-level evidence on the barriers and facilitators on behaviours related to ART among PLHIV using Kaufmans' HIV Behaviour Change model [23]. The secondary objective was to identify evidence gaps for self-reported barriers and facilitators among PLHIV to create lines of action and make recommendations for future research, policy, and practice.

\section{Step 2: identifying a theoretical framework or model}

The complexities and interrelatedness of the factors influencing behaviour of PLHIV, including barriers and facilitators, can be found in multiple dimensions for linkage to ART [31], adherence to ART [32-34] and retention in care $[35,36]$. The dimensions within which barriers and facilitators are understood in this overview are based on the Kaufman and colleagues HIV Behaviour Change Model [23]. The framework includes five broad domains, namely: (1) individual factors (includes factors such as knowledge, emotions, motivation, mental health, adverse drug reactions and comorbidities), (2) interpersonal and network factors (includes factors such as relationships, social networks and interpersonal violence, (3) community factors (includes factors such as stigma, peer pressure and cultural norms), (4) institutional and health system factors, (includes factors such as provision of services, service integration and relationships with health care workers), and (5) structural factors (includes factors such as poverty, political context and gender equity). This framework is useful to this overview as it provides comprehensive multi-level domains to understand the barriers and facilitators that PLHIV experience when they decide to link to ART, adhere to ART and engage in care consistently.

\section{Step 3: criteria for considering systematic reviews for inclusion}

Types of reviews 


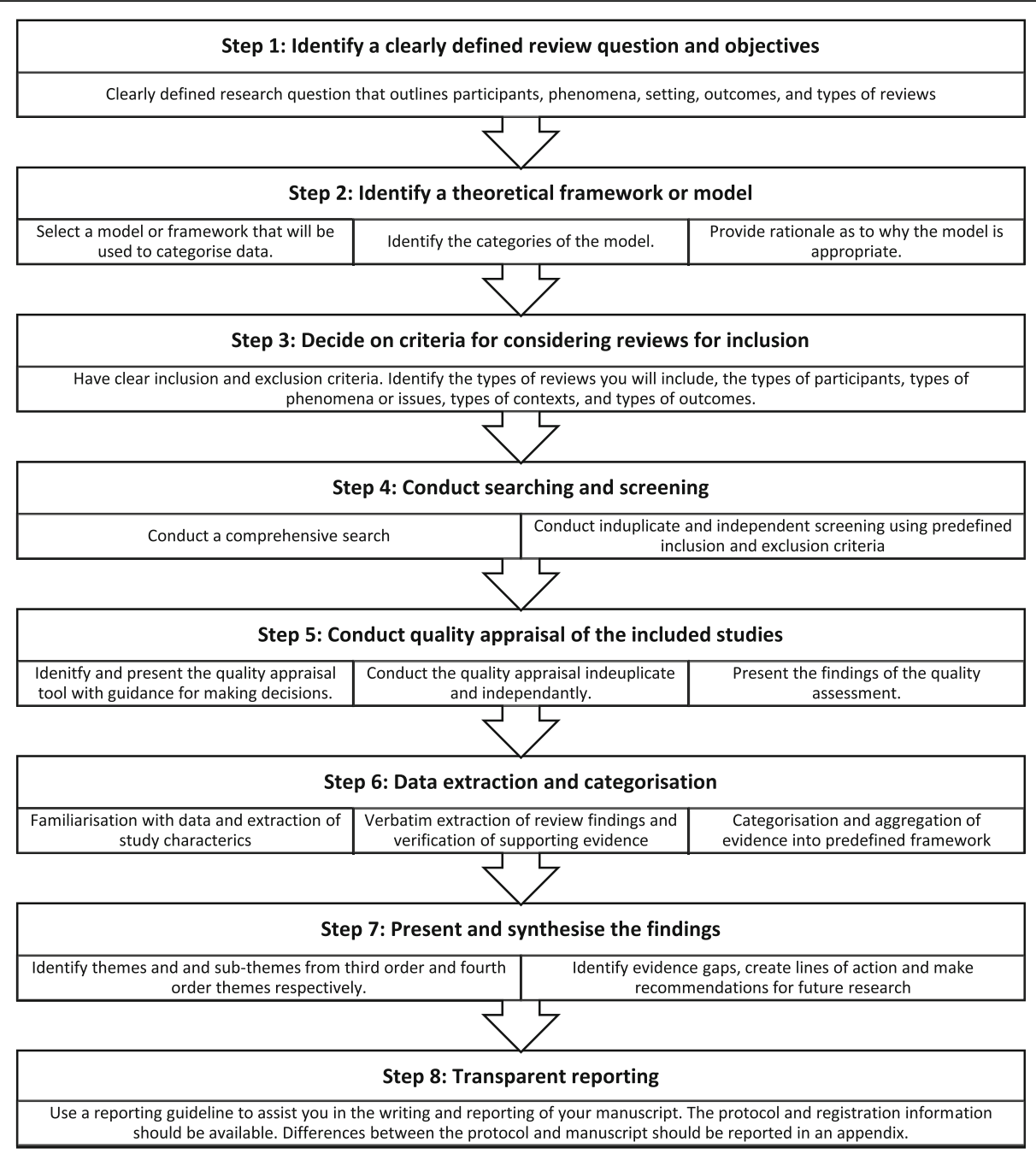

Fig. 1 Steps of the overview using mega-aggregation framework synthesis of qualitative systematic reviews

Systematic reviews were defined as those reviews that had predetermined objectives, predetermined criteria for eligibility, searched at least two data sources, of which one needed to be an electronic database, and performed standardised data extraction [37].

Systematic reviews were considered eligible if they included only qualitative studies. Reviews containing qualitative and quantitative studies were still considered eligible if outcomes were self-reported and a narrative description was used to summarise review findings. Systematic reviews only synthesising quantitative studies or only examining adherence pre- or post-exposure prophylaxis were excluded. No reviews were excluded based on whether quality assessments were conducted or not.

\section{Types of participants}

Eligible participants included children and adults living with HIV. Reviews were excluded if the primary sample of interest included more than $50 \%$ of the population who were not HIV positive. Although PLHIV were the target participants in this review, information obtained from health professionals and primary caregivers were considered if it pertained to perceptions of barriers and facilitators to linkage, adherence and retention in care for PLHIV. Children and youth referred to PLHIV up to the age of 24 years. Where included reviews specifically referred to the age group as youth, older than 12 years, we reported it as youth in our findings.

\section{Types of issues}

Eligible reviews addressed linkage to ART, adherence to treatment and retention in care of persons testing positive for HIV. Enrolment in antiretroviral therapy (ART) care following a positive HIV test is referred to as linkage to care in this overview. While no specific criterion exists with regard to linkage to care, it has been previously defined as one visit or more during the first 6 months of receiving a positive diagnosis and the initiation of antiretroviral treatment [31]. Adherence to ART 
refers to the extent to which a person who is HIV positive follows their prescribed regimen of care and takes their medication as they should [33,34]. Since the introduction of ART, there has been a decline in AIDSrelated deaths and life expectancy for those infected with HIV has increased [15]. Viral suppression is optimal when PLHIV have an adherence rate of $95 \%$ or more [16]. Retention in HIV care is described as constant attainment of the suitable medical care that includes attending follow-up appointments, medical tests or any other activity that was suggested by a healthcare practitioner to be maintained [35]. Reviews addressing the issues related to prevention including pre-exposure prophylaxis (PREP) and pre-ART care were excluded from this overview.

Types of contexts

Reviews synthesising information from high-, middleand low-income countries were included in this overview. The geographic settings included rural and urban across all global regions.

\section{Types of outcomes}

The review-level outcomes of interest were selfreported barriers and facilitators to linkage to ART, adherence to ART and retention in care. Outcomes that were measured and reported using statistical associations between various factors and linkage, adherence and retention in care were not included.

\section{Step 4: conduct searching and screening}

A comprehensive search for systematic reviews up to 25 July 2018 was conducted in the Cochrane Library (specifically the CDSR and DARE), The Campbell Library, MEDLINE via PubMed, SCOPUS and CINAHL EBSCHOhost. PROSPERO was also checked for ongoing systematic reviews. Experts in the field were contacted and reference lists of included reviews were checked to identify further potential reviews for inclusion. An additional search on Google Scholar was conducted to search for reviews not contained within the databases. Key terms included in the search strategy were 'HIV', 'linkage', 'adherence', 'retention in care', 'ART', 'qualitative' and 'systematic reviews'. Search terms were modified appropriately for the various databases. Detailed search strategies for all databases are reported in Additional file 1 . No language, geographic or time restrictions were used in the search. Two authors (LH and AR), using Covidence [38], independently and in duplicate screened titles and abstracts of the records retrieved by the electronic searches for relevance; based on the participant characteristics, issues addressed, study design and outcomes. Full texts were retrieved for all potentially eligible reviews and were screened independently and in duplicate by two authors (LH and AR). Disagreements were recorded in Covidence [38], and these were resolved by consensus or through discussion with a third author (IEW). Reviews were categorised as included, ongoing, awaiting assessment or excluded with reasons.

\section{Step 5: conduct quality appraisal of the included studies}

Included systematic reviews were subjected to quality appraisal by the first author (LH) and second author (AR) independently and in duplicate. Discrepancies were resolved through discussion. Risk of bias was assessed using an amended version of the Joanna Briggs Institute Critical Appraisal Checklist for Systematic Reviews [39] (JBI-SR-Checklist) (Table 1). The JBI-SR-Checklist contains 11 guidance questions for the appraisal of systematic reviews. As this tool can be used for quantitative or qualitative reviews, we only considered those guidance questions that were appropriate for the assessment of qualitative reviews. Therefore, we omitted the question 'Was the likelihood of publication bias assessed?', as this was not applicable to this overview. Furthermore, we added a question that we thought was important to consider, namely 'Was the screening and study selection appropriate?'. Each question was answered as 'yes', 'no' or 'unclear'. The critical appraisal guide [39] provides key considerations for review authors when conducting appraisal. For the purpose of this overview, specific decision rules from the original JBI-SR-Checklist manual [39] were revised (Additional file 2) and clarified for making judgements about risk of bias, in order to ensure consistency between reviewers and across included reviews. No study was excluded based on the results of the quality assessment but rather it was used to identify weaknesses in study methodologies and to strengthen

Table 1 Revised Joanna Briggs Institute (JBI) 11-item checklist for systematic reviews

Revised JBI systematic review checklist items [39]
1. Is the review question clearly and explicitly stated?*
2. Were the inclusion criteria appropriate for the review question?*
3. Was the search strategy appropriate?*
4. Were the sources and resources used to search for studies
adequate?*
5. Was the screening and study selection appropriate?*
6. Were the criteria for appraising studies appropriate?*
7. Was critical appraisal conducted by two or more reviewers
independently?*
8. Were there methods to minimise errors in data extraction?*
9. Were the methods used to combine studies appropriate?*
10. Were recommendations for policy and/or practice supported by the
reported data?
11. Were the specific directives for new research appropriate?

*tems used in the calculation of quality assessment score 
and inform the interpretation of the results of the systematic reviews.

We assessed the overall quality of systematic reviews as either low, medium or high, by considering items 19. Although the area of quality assessment in QES is still being debated in the field and the philosophical underpinning and epistemological reasoning behind conducting or not conducting quality assessment are unique to the rationale and question of the review authors [25], we included these in our assessment. We assessed items 10 and 11, but excluded them from our calculation for level of quality, as these questions do not relate to risk of bias, but rather to the validity of the findings, as stated in the JBI-SR-Checklist manual [39]. Additional File 2 explains how we made decisions about the overall quality of included reviews.

\section{Step 6: data extraction and categorisation}

The data extraction took place in two phases: (1) data extraction of characteristics of included studies and (2) data extraction of barriers and facilitators for data synthesis.

Data extraction of characteristics of included reviews and their primary studies

Data was extracted by the first author (LH) and checked and validated by a second author (AR) and third author (IEW) using a pre-specified piloted data extraction form in Microsoft Excel (Additional file 3). The extracted data included information on databases searched, date of the last search, what the reviews authors searched for and what they found in terms of types of studies, types of participants, the issue of interest, the setting or context, barriers and facilitators related to issues of interest. Details of critical appraisal tools, theoretical frameworks or models, methods of synthesis and limitations were also extracted. Information about the primary studies in the included systematic reviews were extracted, and these included the author names, year of publication, countries included and types of participants from primary source studies relevant to the overview, in order to describe the overlap of primary studies in systematic reviews included in the overview. Review authors were contacted for the full text papers if they were not available to the review team. Discrepancies in data extraction were discussed and once consensus was reached, the second phase commenced.

Data extraction of barriers and facilitators for data synthesis

The first author (LH) read the systematic reviews several times to become more familiar with the findings and recommendations made by the review authors. Following this, LH extracted barriers and facilitators verbatim into Excel for each review and categorised them according to the pre-specified dimensions of the Kaufman HIV Behaviour Change Model [23]. The reviewlevel findings had to be supported by evidence such as references to the primary studies, direct quotes, visual or text evidence from the primary study, visual representations such as tables and figures with references to the primary studies, to be included in the extraction. The second author (AR) and third author (IEW) checked and validated the extracted barriers and facilitators in the Excel spreadsheet, and where discrepancies were raised, consensus was reached through discussion.

\section{Step 7: present and synthesise the findings}

The principles of meta-aggregation and framework synthesis were integrated to design and apply the novel approach of 'mega-aggregative framework synthesis' to this overview. Meta-aggregation is a method of data synthesis used in QES and focuses on aggregating primary-level findings into categories and then further aggregating those categories into synthetic statements that may be used for policy and practice without losing the critical interpretive value of the qualitative findings [21]. Megaaggregation, which is a review-level higher, is a method of qualitative synthesis and aims to aggregate third-order review-level data into higher-order themes, called fourth-order themes with the purpose of identifying the scope of the available review-level evidence and make recommendations for research, policy and practice. In keeping with recent guidelines in selection of approaches for meta-synthesis and the large number of existing reviews available on the topic of this overview, a framework was applied to the mega-aggregative approach. Using a broad framework in mega-aggregation is useful for categorising the themes and findings of systematic review papers which, although may have included various qualitative designs, consider the same objective or issue and outcomes.

As with mega-ethnography [8], this type of synthesis considers first-order constructs (from the person), second-order constructs (interpretations of the author in a primary study), third-order constructs (findings in a systematic review) and fourth-order constructs (findings in an overview of reviews). Using the extracted third-order constructs in the framework dimensions, we then discussed and created fourth-order themes. The third-order concepts were coded into fourth-order concepts further categorised into the appropriate framework dimensions for each of the outcomes. We were then able to review the tables and identify evidence gaps and lines of action to inform future research, policy and practice. The overall number of findings contributing to each of the fourth-order themes of the overview was examined, and the most emergent (meaning the fourth-order 
themes with the most findings) barriers and facilitators, across included systematic reviews, were discussed in the manuscript. Evidence of all findings are presented in in-text tables and within the additional files of the manuscript. Additionally, we identified the evidence gaps and explored the gaps by country income classification, population group and fourthorder themes. Further detail on the application of mega-aggregation framework synthesis to this overview is provided in Additional file 4.

\section{Step 8: transparent reporting}

This overview used guidance from the Johanna Briggs Institute Methodology for Umbrella Reviews [39] and the PRISMA Extension for Scoping Reviews Checklist (PRISMA-ScR) [40] (Additional file 5). The protocol [17] pertaining to this overview was registered on PROSPERO (CRD42017078155) on 17 December 2017. Differences between the protocol and the manuscript are reported in Additional file 6 .

\section{Results}

\section{Overview of the search results}

The database search resulted in 2762 article citations and an additional seven reviews were identified through other sources (two within the reference lists of included reviews and five through other readings). After the removal of duplicates, 1921 citations were imported into Covidence and the title and abstracts were screened, resulting in 78 retrieved for full text review. Thirty-nine reviews were excluded (Additional file 7), most reviews did not fit the criteria of a systematic review $(n=10)$, did not contain qualitative primary studies or data $(n=4)$ or did not include the target population group $(n=1)$. We were unable to obtain the full texts for two reviews and are waiting on information from one author and have classified these three reviews as 'awaiting assessment'. Three ongoing reviews or protocols were found in our search (Additional file 7). We included 33 [41-73] systematic reviews in this overview. Figure 2 describes the flow of reviews through the different stages of this overview using the PRISMA flow diagram [74].

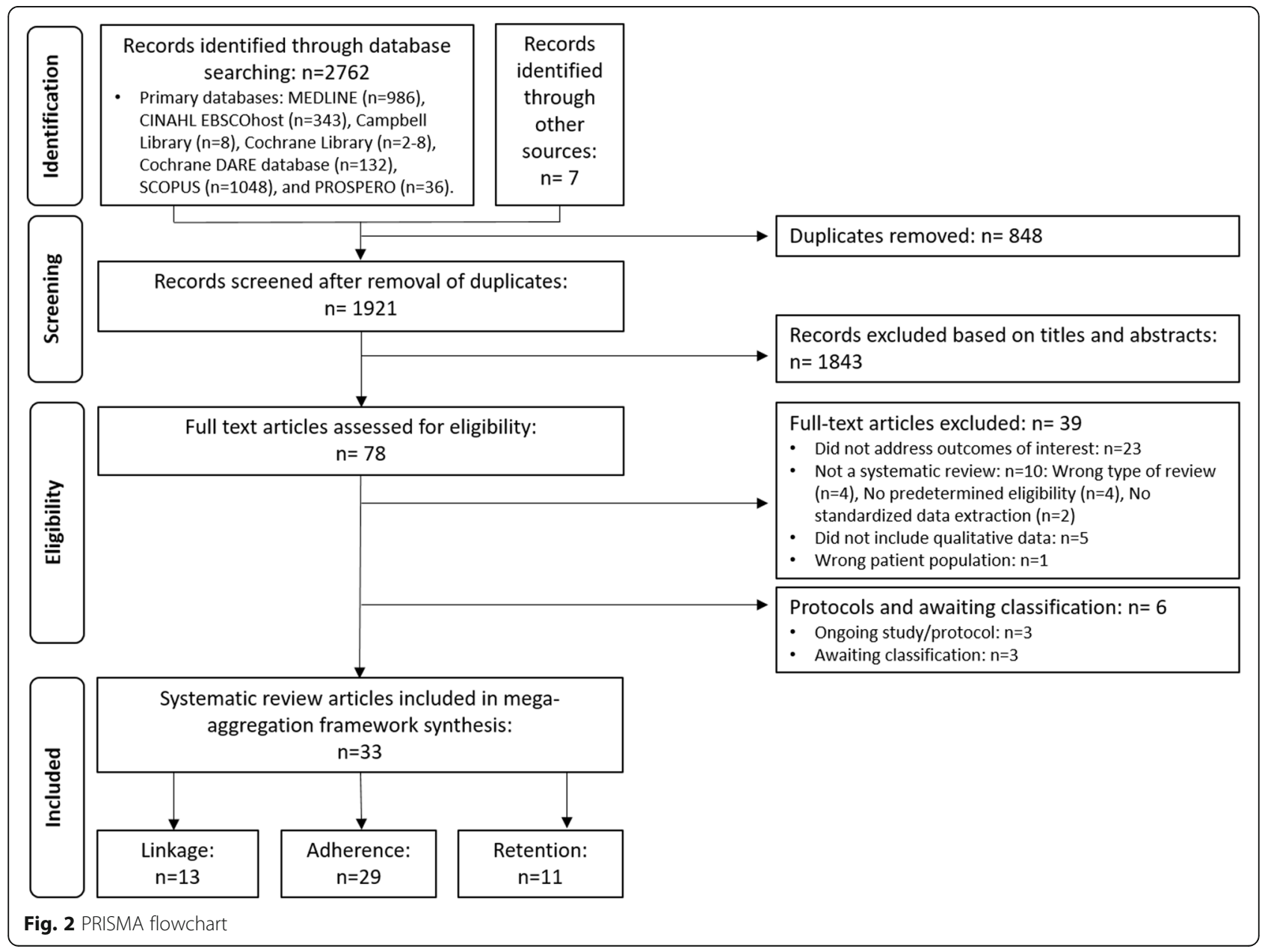




\section{Description of the systematic reviews included in the overview}

Included systematic reviews were published between 2006 to June 2018, peaking at 6 publications in 2018 (Fig. 3).

The included reviews $(N=33)$ synthesised primary studies that were conducted in both high-income countries and lowand middle-income countries with a large concentration of included primary studies being conducted in sub-Saharan Africa (Fig. 4). No reviews written in languages other than English were found.

Self-reported barriers and facilitators of 1,156,540 PLHIV (children and youth, and adults) are included in this overview. Some reviews included high-risk populations, such as pregnant and postpartum women, children and adolescents, commercial sex workers, men who have sex with men, transgender persons, prisoners, intravenous drug users and foreign nationals. Two reviews on children and adolescents included data from caregivers. Table 2 summarises the characteristics of included studies.

We applied the conceptual definitions of the outcomes as per the overview protocol and we found that 13 reviews addressed the outcome of linkage to ART, 29 addressed the outcome of adherence to ART and 11 addressed the outcome of retention in care (see Fig. 2). The method of synthesis of the reviews varied and included thematic analysis, thematic content analysis, content analysis, narrative synthesis, meta-synthesis and meta-aggregation. Details of data are extracted, and critical appraisal of each included review is available in Additional file 8 .

Due to the different ways of reporting results in the included reviews, we discerned between two population groups in terms of age, children and youth, and adults.

\section{Overlap between included systematic reviews}

We found overlap in the qualitative, quantitative and mixed methods primary studies included within the 33 systematic reviews (Additional file 9). Review authors used varying definitions of linkage to ART, adherence to ART and retention in care when considering studies for inclusion. One author may have used a primary study to synthesise evidence on linkage to ART and another author may have used the same study to synthesis evidence on adherence to ART. The primary studies within the systematic reviews were published between and 1995 and 2017. Of the 1153 primary studies in the systematic reviews, 826 were unique studies, of which 616 were included in only one review. We found that 139 of the studies were included in two reviews, forty-seven in three reviews, fourteen in four reviews, three in five, four in six, and one study was included in seven reviews and another across eight reviews.

We explored whether there was overlap in the search dates between the included reviews and found that most reviews searched between 2000 and 2013 with an average search period covering 13 years (Fig. 5). Eight studies $[44,45,56,58,60,65,67,71]$ conducted comprehensive searches up to a year before publication. One review [41], conducted as part of an online postgraduate degree programme, had very short search period of 6 months. We found considerable overlap in the search dates of included systematic reviews.

\section{Quality assessment of systematic reviews included in the review}

The methodological quality of the included systematic reviews varied across reviews. Details of the justifications for quality judgements are reported in Additional file 8.

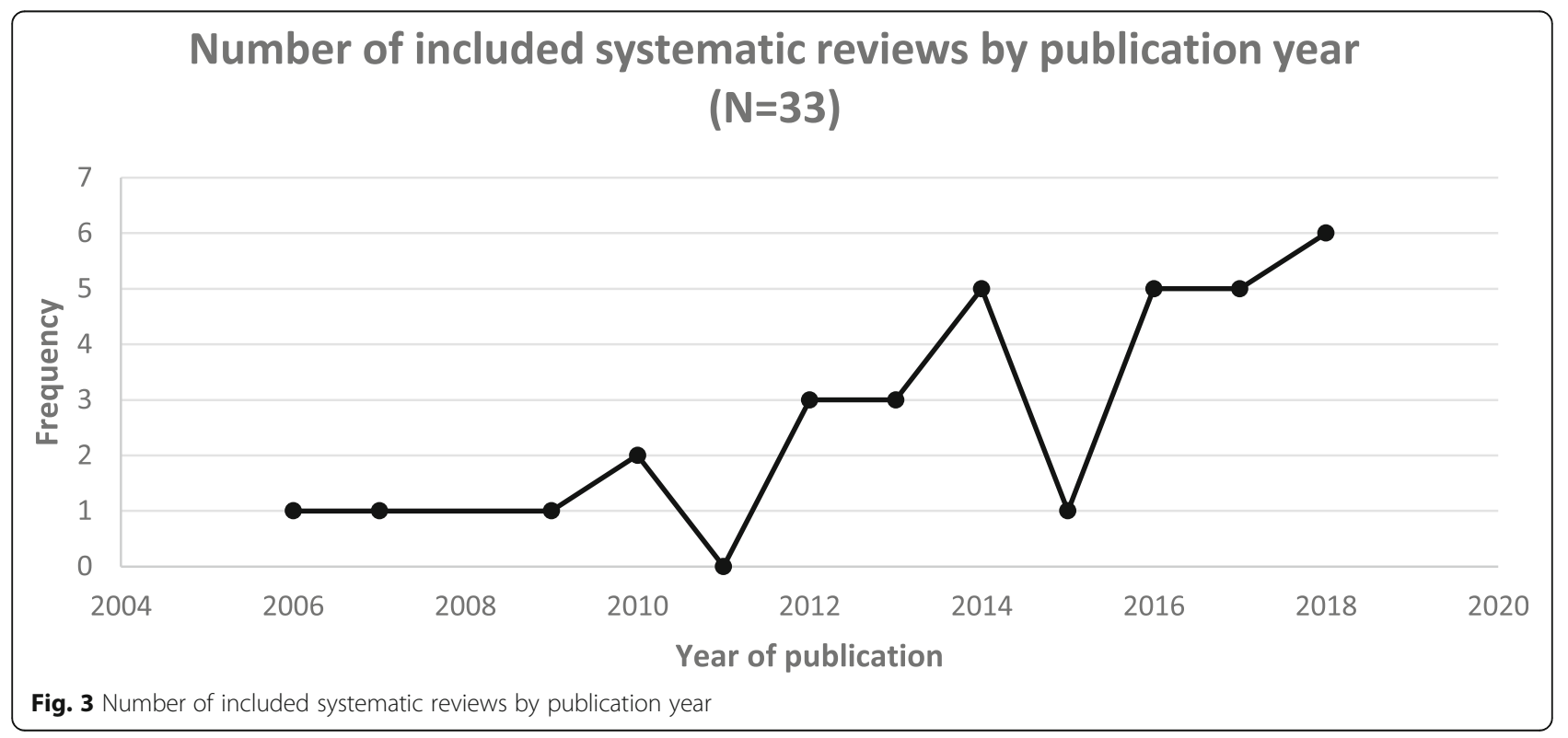




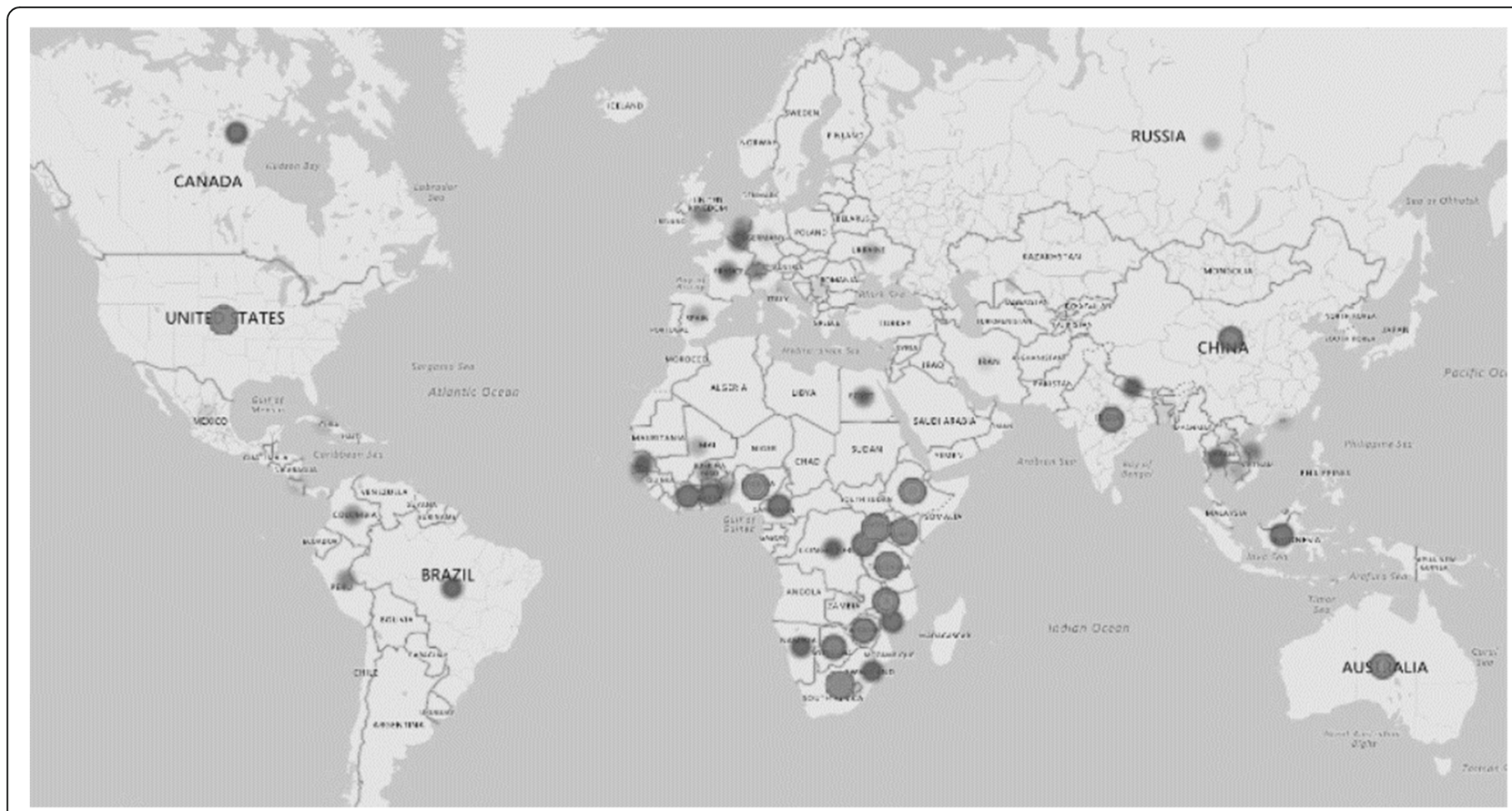

Fig. 4 Distribution of countries included in the included systematic reviews ( $N=33$ )

All but five reviews had clear research questions. Key methodological aspects that were appraised as good quality were the relevance of recommendations for policy, having a clear research question and relevant directives for future research. The key aspects that were assessed as poor for the included reviews were the sources used to search and the inclusion criteria of the reviews. Some reviews did not clearly report items, and as we were thus unable to make a judgement, we assessed them as 'unclear'. Items concerning if the 'process of data extraction was appropriate' and if the 'critical appraisal was conducted by two or more authors' were mostly identified as 'unclear' across included reviews. We identified 110 (30.3\%) items out a possible 363 as 'unclear', 70 (19.3\%) items as 'no' and 183 (50.4\%) of items as 'yes'. Two reviews $[62,65]$ were rated as high quality, six reviews $[41,43,46$, $55,61,67]$ were rated as medium quality and 25 reviews $[42,44,45,47-54,56-60,63,66,68-73,75]$ were rated as low quality (Table 3 ).

\section{Data categorisation: what is the available review-level evidence on barriers and facilitators to linkage, adherence, and retention in care?}

We found 544 unique third-order concepts from the included systematic reviews and to retain the essence of the review authors' interpretations, we extracted concepts verbatim. We then categorised and aggregated the evidence into the predetermined framework, namely, Kaufman's Behaviour Change Model of HIV [23].

\section{Barriers and facilitators to linkage to $A R T$}

Barriers and facilitators to linkage to ART were found on all levels of the Kaufman framework (Additional file 10) and contributed to the synthesis of the barriers and facilitators to linkage to ART. One low-quality review [73] contributed to the findings on linkage for children. For adults, findings for linkage, were aggregated from one high-quality review [65], three medium-quality review $[43,46,55]$, and eleven low-quality reviews $[42,44$, $45,49,50,53,54,58,60,64,66]$.

On the individual level, participants reported barriers linked to sociodemographic factors (5 findings), such as being younger, whether the participant's occupation was considered socially acceptable, gender, and not having identification documents in order to enrol in care services. Barriers related to patient fears (9 findings) were the emergent themes. PLHIV expressed fears of the consequences of disclosure, such as job loss, stigma and social isolation, fears of being on lifelong treatment and the negative side effects of ART. PLHIV reported experiencing psychological distress and emotional reactions (9 findings) and some were shocked at the news of their positive status, unsure about how they had contracted the disease, and the possibility of infidelity in their relationships. Feelings of hopelessness and depression were a recurring theme, with women questioning their selfesteem as wives and mothers. For children, two findings of barriers to linkage included negative emotions and self-perception. 
Table 2 Table of included studies ( $N=33$ )

\begin{tabular}{|c|c|c|c|c|c|c|c|c|}
\hline \multirow{2}{*}{$\begin{array}{l}\text { First author, } \\
\text { year of } \\
\text { publication } \\
\text { [reference] }\end{array}$} & \multirow{2}{*}{$\begin{array}{l}\text { Search } \\
\text { dates }\end{array}$} & \multirow[t]{2}{*}{ Participants } & \multirow[t]{2}{*}{ Issue } & \multicolumn{2}{|l|}{ Context } & \multirow{2}{*}{$\begin{array}{l}\text { Types of } \\
\text { studies }\end{array}$} & \multirow{2}{*}{$\begin{array}{l}\text { Method of } \\
\text { synthesis }\end{array}$} & \multirow{2}{*}{$\begin{array}{l}\text { Overall } \\
\text { quality } \\
\text { of } \\
\text { review }\end{array}$} \\
\hline & & & & $\begin{array}{l}\text { Low- to middle- } \\
\text { income country }\end{array}$ & $\begin{array}{l}\text { High-income } \\
\text { country }\end{array}$ & & & \\
\hline $\begin{array}{l}\text { Ammon, } 2018 \\
{[41]}\end{array}$ & $\begin{array}{l}3 \text { June } 2016 \\
\text { to } 15 \\
\text { August } \\
2016\end{array}$ & $\begin{array}{l}N=3145 \\
\text { participants: } 2937 \\
\text { adolescents aged } \\
\text { 10-19; } 191 \text { care- } \\
\text { givers (parents, non- } \\
\text { parental caregiver, } \\
\text { biological relative, } \\
\text { non-relative, or } \\
\text { foster-carer) and } 17 \\
\text { healthcare providers. } \\
\text { Some adolescents } \\
\text { living with HIV did } \\
\text { not know about } \\
\text { their HIV-positive } \\
\text { status. }\end{array}$ & Adherence & $\begin{array}{l}\text { Sub-Saharan Africa: } \\
n=1 \text { study each } \\
\text { from Congo DRC, } \\
\text { Ghana, Kenya, } \\
\text { Rwanda, South } \\
\text { Africa, Zambia, } \\
\text { Zimbabwe, and } n= \\
2 \text { studies from } \\
\text { Uganda. }\end{array}$ & None & $\begin{array}{l}\text { 11 studies: } \\
\text { Qualitative } \\
\text { (7), } \\
\text { Quantitative } \\
\text { (1) and } \\
\text { Mixed } \\
\text { Methods (3) }\end{array}$ & $\begin{array}{l}\text { Thematic } \\
\text { synthesis }\end{array}$ & Medium \\
\hline $\begin{array}{l}\text { Barroso, } 2017 \\
\text { [42] }\end{array}$ & $\begin{array}{l}2008 \text { to } \\
2013\end{array}$ & $\begin{array}{l}N=6189 \\
\text { participants: } n= \\
4830 \text { PLHIV (2197 } \\
\text { female and } 1850 \\
\text { male, } 783 \\
\text { unspecified) and } n \\
=1359 \text { included } \\
\text { provider participants } \\
\text { (caregivers, health } \\
\text { care providers, } \\
\text { traditional healers, } \\
\text { local community } \\
\text { leaders, pharmacists, } \\
\text { policymakers, } \\
\text { stakeholders, peer } \\
\text { counsellors, facility } \\
\text { managers, } \\
\text { volunteers, and } \\
\text { clinical trial } \\
\text { coordinators). }\end{array}$ & $\begin{array}{l}\text { Linkage } \\
\text { Adherence }\end{array}$ & $\begin{array}{l}\text { China (5), Nigeria (5), } \\
\text { South Africa (19), } \\
\text { Tanzania (8), } \\
\text { Uganda (16), and } \\
\text { Zambia (9). All other } \\
\text { locations for data } \\
\text { collection } \\
\text { contributed to } \\
\text { fewer than five } \\
\text { reports (Countries } \\
\text { not reported) }\end{array}$ & Europe (9), US (28) & $\begin{array}{l}\text { 127 studies: } \\
\text { Qualitative } \\
\text { (127) }\end{array}$ & $\begin{array}{l}\text { Thematic } \\
\text { synthesis }\end{array}$ & Low \\
\hline $\begin{array}{l}\text { Bolsewicz, } \\
2015 \text { [43] }\end{array}$ & $\begin{array}{l}2003 \text { to } \\
2013\end{array}$ & $\begin{array}{l}\text { PLHIV, excluding } \\
\text { drug users, mothers, } \\
\text { adolescents, } \\
\text { prisoners, sex } \\
\text { workers in Canada, } \\
\text { UK, and Australia }\end{array}$ & $\begin{array}{l}\text { Linkage } \\
\text { Adherence }\end{array}$ & None & $\begin{array}{l}\text { Canada (8), UK (3) } \\
\text { and Australia (6) }\end{array}$ & & $\begin{array}{l}\text { Thematic } \\
\text { synthesis }\end{array}$ & Medium \\
\hline $\begin{array}{l}\text { Bravo, } 2010 \\
{[44]}\end{array}$ & $\begin{array}{l}1990 \text { to } \\
\text { November } \\
2009\end{array}$ & $\begin{array}{l}N=4215 \text { PLHIV } \\
\text { including drug users } \\
\text { and women caring } \\
\text { for children }<18 \\
\text { years; } n=4022 \text { in } \\
\text { Quantitative and } n \\
=193 \text { Qualitative } \\
\text { studies. }\end{array}$ & $\begin{array}{l}\text { Linkage } \\
\text { Adherence }\end{array}$ & Botswana (1) & $\begin{array}{l}\text { US (7), UK (1), } \\
\text { France (1) }\end{array}$ & $\begin{array}{l}10 \text { studies: } \\
\text { Qualitative } \\
\text { (5) and } \\
\text { Quantitative } \\
\text { (5) }\end{array}$ & $\begin{array}{l}\text { Thematic } \\
\text { meta- } \\
\text { analysis }\end{array}$ & Low \\
\hline $\begin{array}{l}\text { Chop, } 2017 \\
{[45]}\end{array}$ & $\begin{array}{l}\text { Up to } 18 \\
\text { February } \\
2018\end{array}$ & $\begin{array}{l}\text { Women living with } \\
\text { HIV }\end{array}$ & Adherence & $\begin{array}{l}\text { Zambia (1), } \\
\text { Swaziland (1) and } \\
\text { Democratic Republic } \\
\text { of Congo (1) }\end{array}$ & France (1) & $\begin{array}{l}4 \text { studies: } \\
\text { Qualitative } \\
\text { (3) and } \\
\text { Quantitative } \\
\text { (1) }\end{array}$ & $\begin{array}{l}\text { Thematic } \\
\text { analysis }\end{array}$ & Low \\
\hline $\begin{array}{l}\text { Colvin, } 2014 \\
\text { [46] }\end{array}$ & $\begin{array}{l}1 \text { January } \\
2008 \text { to } 26 \\
\text { March } 2013\end{array}$ & $\begin{array}{l}N=875308 \\
\text { participants: HIV- } \\
\text { infected pregnant } \\
\text { and/or postpartum } \\
\text { women and/or } \\
\text { health care pro- } \\
\text { viders delivering } \\
\text { antenatal care, ART }\end{array}$ & $\begin{array}{l}\text { Linkage } \\
\text { Adherence } \\
\text { Retention }\end{array}$ & $\begin{array}{l}\text { Sub-Saharan Africa } \\
\text { (38), Latin America } \\
\text { (2), and Asia (2) }\end{array}$ & None & $\begin{array}{l}\text { 42 studies: } \\
\text { Qualitative } \\
\text { (14), } \\
\text { Quantitative } \\
\text { studies (25) } \\
\text { and Mixed } \\
\text { Methods (3) }\end{array}$ & $\begin{array}{l}\text { Narrative } \\
\text { meta- } \\
\text { synthesis }\end{array}$ & Medium \\
\hline
\end{tabular}


Table 2 Table of included studies ( $N=33$ ) (Continued)

\begin{tabular}{|c|c|c|c|c|c|c|c|c|}
\hline \multirow{2}{*}{$\begin{array}{l}\text { First author, } \\
\text { year of } \\
\text { publication } \\
\text { [reference] }\end{array}$} & \multirow{2}{*}{$\begin{array}{l}\text { Search } \\
\text { dates }\end{array}$} & \multirow[t]{2}{*}{ Participants } & \multirow[t]{2}{*}{ Issue } & \multicolumn{2}{|l|}{ Context } & \multirow{2}{*}{$\begin{array}{l}\text { Types of } \\
\text { studies }\end{array}$} & \multirow{2}{*}{$\begin{array}{l}\text { Method of } \\
\text { synthesis }\end{array}$} & \multirow{2}{*}{$\begin{array}{l}\text { Overall } \\
\text { quality } \\
\text { of } \\
\text { review }\end{array}$} \\
\hline & & & & $\begin{array}{l}\text { Low- to middle- } \\
\text { income country }\end{array}$ & $\begin{array}{l}\text { High-income } \\
\text { country }\end{array}$ & & & \\
\hline & & $\begin{array}{l}\text { and/or PMTCT. A } \\
\text { few studies included } \\
\text { partners and/or fam- } \\
\text { ily members. }\end{array}$ & & & & & & \\
\hline $\begin{array}{l}\text { Croome, } 2017 \\
\text { [47] }\end{array}$ & $\begin{array}{l}2005 \text { to } 24 \\
\text { May } 2016\end{array}$ & $\begin{array}{l}N=37175 \text { Adult } \\
\text { PLHIV }\end{array}$ & Adherence & $\begin{array}{l}\text { Benin, Cote d'Ivoire } \\
\text { and Mali (1), } \\
\text { Botswana (3), } \\
\text { Burkina Faso (1), } \\
\text { Cameroon (4), Cote } \\
\text { d'Ivoire (1), DRC (2), } \\
\text { Ethiopia (20), } \\
\text { Ethiopia and } \\
\text { Uganda (1), Ghana } \\
\text { (4), Guinea-Bissau } \\
\text { (1), Kenya (16), } \\
\text { Kenya and Malawi } \\
\text { (1), Kenya and } \\
\text { Uganda (1), Lesotho } \\
\text { (1), Malawi (2), Mali } \\
\text { (1), Mozambique (3), } \\
\text { Namibia (4), Nigeria } \\
\text { (13), Nigeria, } \\
\text { Tanzania and } \\
\text { Uganda (1), Rwanda } \\
\text { (3), Senegal (1), } \\
\text { South Africa (30), } \\
\text { Tanzania (10), } \\
\text { Tanzania, Uganda } \\
\text { and Zambia (1), } \\
\text { Togo (1), Uganda } \\
\text { (19), Zambia (6), } \\
\text { Zimbabwe (2) }\end{array}$ & None & $\begin{array}{l}154 \text { studies: } \\
83 \\
\text { Qualitative } \\
\text { (83), } \\
\text { Quantitative } \\
\text { (67) and } \\
\text { Mixed } \\
\text { methods (4) }\end{array}$ & $\begin{array}{l}\text { Thematic } \\
\text { content } \\
\text { analysis }\end{array}$ & Low \\
\hline $\begin{array}{l}\text { Engler, } 2018 \\
\text { [48] }\end{array}$ & $\begin{array}{l}1996 \text { to } 10 \\
\text { March } 2016\end{array}$ & $\begin{array}{l}N=1482 \text { adult } \\
\text { PLHIV (including } \\
\text { men, women, men } \\
\text { who have sex with } \\
\text { men (MSM), } \\
\text { intravenous drug } \\
\text { user (IDU) }\end{array}$ & Adherence & None & $\begin{array}{l}\text { US (35), Europe (3) } \\
\text { (Switzerland, the } \\
\text { Netherlands and } \\
\text { Belgium), and } \\
\text { Canada (2). }\end{array}$ & $\begin{array}{l}40 \text { studies: } \\
\text { Qualitative } \\
(40)\end{array}$ & $\begin{array}{l}\text { Thematic } \\
\text { analysis }\end{array}$ & Low \\
\hline $\begin{array}{l}\text { Ferguson, } \\
2012^{(}[49]^{\prime}\end{array}$ & $\begin{array}{l}\text { 1st January } \\
2000 \text { to } \\
31 \text { st } \\
\text { December } \\
2010\end{array}$ & $\begin{array}{l}N=819 \text { Pregnant } \\
\text { women with HIV. } \\
\text { Not all studies } \\
\text { included reported } \\
\text { sample size. }\end{array}$ & Retention & $\begin{array}{l}\text { Kenya (1), South } \\
\text { Africa (1), Tanzania } \\
\text { (1), Zimbabwe (1), } \\
\text { Malawi (2), Uganda } \\
\text { (1) }\end{array}$ & None & $\begin{array}{l}7 \text { studies: } \\
\text { Qualitative } \\
\text { (3) and } \\
\text { Quantitative } \\
\text { (4) }\end{array}$ & $\begin{array}{l}\text { Thematic } \\
\text { content } \\
\text { analysis }\end{array}$ & Low \\
\hline $\begin{array}{l}\text { Flores, } 2018 \\
\text { [50] }\end{array}$ & $\begin{array}{l}2008 \text { to } \\
2013\end{array}$ & $\begin{array}{l}N=3257 \\
\text { participants: } 2263 \\
\text { PLHIV (740 men, } \\
1008 \text { women, } 78 \\
\text { transgender } \\
\text { individuals and } 437 \\
\text { people with } \\
\text { unspecified gender). } \\
994 \text { other people } \\
\text { were included in } \\
\text { the studies such as } \\
\text { family members, } \\
\text { friends, physicians, } \\
\text { nurses, treatment } \\
\text { advocates, } \\
\text { caregivers, clinic } \\
\text { staff, programme } \\
\text { directors, social } \\
\text { workers, and other }\end{array}$ & $\begin{array}{l}\text { Linkage } \\
\text { Retention }\end{array}$ & $\begin{array}{l}\text { South Africa (9), } \\
\text { Uganda (6), Nigeria } \\
\text { (4), Zimbabwe (4) } \\
\text { and China (4); } 20= \\
\text { unspecified }\end{array}$ & US (22 reports) & $\begin{array}{l}\text { 69 studies: } \\
\text { Qualitative } \\
\text { (69) }\end{array}$ & $\begin{array}{l}\text { Thematic } \\
\text { meta- } \\
\text { synthesis }\end{array}$ & Low \\
\hline
\end{tabular}


Table 2 Table of included studies ( $N=33$ ) (Continued)

\begin{tabular}{|c|c|c|c|c|c|c|c|c|}
\hline \multirow{2}{*}{$\begin{array}{l}\text { First author, } \\
\text { year of } \\
\text { publication } \\
\text { [reference] }\end{array}$} & \multirow{2}{*}{$\begin{array}{l}\text { Search } \\
\text { dates }\end{array}$} & \multirow[t]{2}{*}{ Participants } & \multirow[t]{2}{*}{ Issue } & \multicolumn{2}{|l|}{ Context } & \multirow{2}{*}{$\begin{array}{l}\text { Types of } \\
\text { studies }\end{array}$} & \multirow{2}{*}{$\begin{array}{l}\text { Method of } \\
\text { synthesis }\end{array}$} & \multirow{2}{*}{$\begin{array}{l}\text { Overall } \\
\text { quality } \\
\text { of } \\
\text { review }\end{array}$} \\
\hline & & & & $\begin{array}{l}\text { Low- to middle- } \\
\text { income country }\end{array}$ & $\begin{array}{l}\text { High-income } \\
\text { country }\end{array}$ & & & \\
\hline & & key stakeholders. & & & & & & \\
\hline $\begin{array}{l}\text { Gaston, } 2013 \\
\text { [51] }\end{array}$ & $\begin{array}{l}1 \text { January } \\
2001 \text { to } 31 \\
\text { May } 2012\end{array}$ & $\begin{array}{l}\text { African Americans } \\
\text { LHIV Total } n=2846\end{array}$ & Adherence & None & USA (16) & $\begin{array}{l}16 \text { Studies: } \\
\text { Qualitative } \\
\text { (6) and } \\
\text { Quantitative } \\
\text { (10) }\end{array}$ & $\begin{array}{l}\text { Thematic } \\
\text { analysis }\end{array}$ & Low \\
\hline $\begin{array}{l}\text { Geter, } 2018 \\
\text { [52] }\end{array}$ & $\begin{array}{l}\text { January } \\
2005 \text { to } \\
\text { December } \\
2016\end{array}$ & $\begin{array}{l}\text { African American } \\
\text { females living with } \\
\text { HIV Total } n=830\end{array}$ & $\begin{array}{l}\text { Adherence } \\
\text { Retention }\end{array}$ & None & US (14) & $\begin{array}{l}14 \text { studies: } \\
\text { Qualitative } \\
\text { (10) and } \\
\text { Quantitative } \\
\text { (4) }\end{array}$ & $\begin{array}{l}\text { Thematic } \\
\text { content } \\
\text { analysis }\end{array}$ & Low \\
\hline $\begin{array}{l}\text { Govindasamy, } \\
2012 \text { [53] }\end{array}$ & $\begin{array}{l}01 \text { January } \\
2000 \text { to } 31 \\
\text { May } 2011\end{array}$ & $\begin{array}{l}\text { PLHIV in sub- } \\
\text { Saharan Africa and } \\
\text { health care workers. }\end{array}$ & Linkage & $\begin{array}{l}\text { South Africa (6), } \\
\text { Uganda (6), Kenya } \\
(2), \text { Tanzania (2), } \\
\text { Zambia (2), and } 1 \\
\text { study each from } \\
\text { Ethiopia, Swaziland, } \\
\text { Mozambique, and } \\
\text { South Africa and } \\
\text { Zimbabwe. }\end{array}$ & None & $\begin{array}{l}\text { 21 Studies: } \\
\text { Qualitative } \\
\text { (11), } \\
\text { Quantitative } \\
\text { (7) and } \\
\text { Mixed } \\
\text { Methods (3) }\end{array}$ & $\begin{array}{l}\text { Thematic } \\
\text { content } \\
\text { analysis }\end{array}$ & Low \\
\hline $\begin{array}{l}\text { Heestermans, } \\
2016 \text { [54] }\end{array}$ & $\begin{array}{l}\text { January } \\
2002 \text { to } 27 \\
\text { October } \\
2014 .\end{array}$ & 161922 Adult PLHIV & Adherence & Sub-Saharan Africa & & $\begin{array}{l}\text { 146 studies: } \\
\text { Qualitative } \\
\text { (37), } \\
\text { Quantitative } \\
\text { (112) and } \\
\text { Mixed } \\
\text { methods (3) }\end{array}$ & $\begin{array}{l}\text { Narrative } \\
\text { synthesis }\end{array}$ & Low \\
\hline $\begin{array}{l}\text { Hodgson, } \\
2014 \text { [55] }\end{array}$ & $\begin{array}{l}\text { 1st January } \\
2008 \text { to } 26 \\
\text { March } 2013\end{array}$ & $\begin{array}{l}\text { Pregnant women } \\
\text { and postpartum } \\
\text { women infected } \\
\text { with HIV }\end{array}$ & $\begin{array}{l}\text { Linkage } \\
\text { Adherence } \\
\text { Retention }\end{array}$ & $\begin{array}{l}\text { Ghana (1), Nigeria } \\
\text { (1), Malawi (5), } \\
\text { South Africa (6), } \\
\text { Zimbabwe (2), } \\
\text { Tanzania (2), Kenya } \\
\text { (5), Uganda (3), } \\
\text { Brazil (1), Rwanda } \\
\text { (1), Zambia (1), Latin } \\
\text { America (1) }\end{array}$ & $\begin{array}{l}\text { Australia (1), US (3), } \\
\text { France (1), }\end{array}$ & $\begin{array}{l}34 \text { studies } \\
\text { included in } \\
\text { the review: } \\
\text { Qualitative } \\
\text { (12), } \\
\text { Quantitative } \\
\text { (16) and } \\
\text { Mixed } \\
\text { Methods (6) }\end{array}$ & $\begin{array}{l}\text { Thematic } \\
\text { analysis }\end{array}$ & Medium \\
\hline Katz, 2013 [56] & $\begin{array}{l}\text { Up until } \\
\text { February } \\
2013\end{array}$ & $\begin{array}{l}\text { PLHIV between } 18 \\
\text { and } 30 \text { years old, } \\
\text { providers of HIV } \\
\text { care, single persons } \\
\text { and those in } \\
\text { intimate } \\
\text { partnerships and } \\
\text { persons with and } \\
\text { without children. } \\
\text { High-risk groups in- } \\
\text { cluding men who } \\
\text { have sex with men, } \\
\text { injecting drug users } \\
\text { and commercial sex } \\
\text { workers. }\end{array}$ & Adherence & $\begin{array}{l}\text { Uganda (9), South } \\
\text { Africa (5), India (2), } \\
\text { and } 1 \text { study each } \\
\text { from DRC, Brazil, } \\
\text { Botswana, Tanzania, } \\
\text { Thailand, Egypt, } \\
\text { Ethiopia, Vietnam, } \\
\text { Nepal, Nigeria, Asia, } \\
\text { Zambia, and China. } \\
\text { Four countries were } \\
\text { not reported. }\end{array}$ & US (1) & $\begin{array}{l}75 \text { Studies: } \\
\text { Quantitative } \\
\text { (41) and } \\
\text { Qualitative } \\
\text { (34) }\end{array}$ & $\begin{array}{l}\text { Meta- } \\
\text { ethnography }\end{array}$ & Low \\
\hline $\begin{array}{l}\text { Knettel, } 2018 \\
\text { [57] }\end{array}$ & $\begin{array}{l}\text { January } \\
2012 \text { to } \\
\text { June } 2017\end{array}$ & $\begin{array}{l}736 \text { Pregnant and } \\
\text { postpartum women } \\
\text { on option B+. }\end{array}$ & Retention & $\begin{array}{l}\text { Malawi (13), Uganda } \\
\text { (4), Zimbabwe (3), } \\
\text { Mozambique (2), } \\
\text { and } 1 \text { each from } \\
\text { Cameroon, Ethiopia, } \\
\text { Rwanda, South } \\
\text { Africa, and Tanzania }\end{array}$ & None & $\begin{array}{l}13 \text { Studies: } \\
\text { Qualitative } \\
\text { (13) }\end{array}$ & $\begin{array}{l}\text { Thematic } \\
\text { analysis }\end{array}$ & Low \\
\hline $\begin{array}{l}\text { Lancaster, } \\
2016 \text { [58] }\end{array}$ & $\begin{array}{l}\text { Up to } 22 \\
\text { November } \\
2013 \text { and a }\end{array}$ & $\begin{array}{l}N=2721 \text { Female } \\
\text { sex workers living } \\
\text { with HIV }\end{array}$ & $\begin{array}{l}\text { Linkage } \\
\text { Adherence }\end{array}$ & $\begin{array}{l}\text { Rwanda (1), } \\
\text { Zimbabwe (2), Benin } \\
\text { (2), Burkina Faso (1), }\end{array}$ & None & $\begin{array}{l}10 \text { studies: } \\
\text { Qualitative } \\
\text { (3), }\end{array}$ & $\begin{array}{l}\text { Thematic } \\
\text { analysis }\end{array}$ & Low \\
\hline
\end{tabular}


Table 2 Table of included studies ( $N=33$ ) (Continued)

\begin{tabular}{|c|c|c|c|c|c|c|c|c|}
\hline \multirow{2}{*}{$\begin{array}{l}\text { First author, } \\
\text { year of } \\
\text { publication } \\
\text { [reference] }\end{array}$} & \multirow{2}{*}{$\begin{array}{l}\text { Search } \\
\text { dates }\end{array}$} & \multirow[t]{2}{*}{ Participants } & \multirow[t]{2}{*}{ Issue } & \multicolumn{2}{|l|}{ Context } & \multirow{2}{*}{$\begin{array}{l}\text { Types of } \\
\text { studies }\end{array}$} & \multirow{2}{*}{$\begin{array}{l}\text { Method of } \\
\text { synthesis }\end{array}$} & \multirow{2}{*}{$\begin{array}{l}\text { Overall } \\
\text { quality } \\
\text { of } \\
\text { review }\end{array}$} \\
\hline & & & & $\begin{array}{l}\text { Low- to middle- } \\
\text { income country }\end{array}$ & $\begin{array}{l}\text { High-income } \\
\text { country }\end{array}$ & & & \\
\hline & $\begin{array}{l}\text { second } \\
\text { search up } \\
\text { to } 30 \text { July } \\
2015\end{array}$ & & & $\begin{array}{l}\text { Nigeria (1), } \\
\text { Swaziland (1), Kenya } \\
\text { (1), and Uganda (1). }\end{array}$ & & $\begin{array}{l}\text { Quantitative } \\
\text { (6) and } \\
\text { Mixed } \\
\text { Methods (3) }\end{array}$ & & \\
\hline $\begin{array}{l}\text { Lankowski, } \\
2014 \text { [59] }\end{array}$ & $\begin{array}{l}\text { Databases } \\
\text { up until } \\
\text { August } \\
2011 \text { and } \\
\text { abstracts } \\
\text { from } 2002 \\
\text { to } 2004 \text { and } \\
\text { from } 2006 \\
\text { to } 2011 \text {. }\end{array}$ & $\begin{array}{l}\text { N = } 69506 \text { Adults } \\
\text { and children LHIV, } \\
\text { HIV-infected HCW, } \\
\text { HC Providers, HIV- } \\
\text { infected rape vic- } \\
\text { tims, pregnant and } \\
\text { postpartum women } \\
\text { with HIV. }\end{array}$ & $\begin{array}{l}\text { Linkage } \\
\text { Adherence } \\
\text { Retention }\end{array}$ & $\begin{array}{l}\text { Uganda (10), Kenya } \\
\text { (3), Zambia (2), } \\
\text { Malawi (4), Nigeria } \\
\text { (3), Corte d'Ivoire (1), } \\
\text { Botswana (4), } \\
\text { Tanzania (4), Togo } \\
\text { (1), Ethiopia (1), } \\
\text { South Africa (2), The } \\
\text { Gambia (1), Namibia } \\
\text { (1) }\end{array}$ & None & $\begin{array}{l}34 \text { studies: } \\
\text { Qualitative } \\
\text { (16) and } \\
\text { Quantitative } \\
\text { (18) }\end{array}$ & $\begin{array}{l}\text { Content } \\
\text { analysis }\end{array}$ & Low \\
\hline $\begin{array}{l}\text { Lazuardi, } 2018 \\
\text { [60] }\end{array}$ & $\begin{array}{l}1990 \text { to } \\
2016\end{array}$ & $\begin{array}{l}\text { PLHIV: including } \\
\text { injecting drug users, } \\
\text { pregnant women, } \\
\text { MSM, transgendered } \\
\text { people, women, } \\
\text { men, and sero- } \\
\text { discordant couples. } \\
\text { Found information } \\
\text { related to service } \\
\text { providers, commu- } \\
\text { nity members, TB } \\
\text { patients, caregivers, } \\
\text { and community } \\
\text { organisers. }\end{array}$ & $\begin{array}{l}\text { Linkage } \\
\text { Adherence } \\
\text { Retention }\end{array}$ & Indonesia (11) & None & $\begin{array}{l}11 \text { studies: } \\
\text { Qualitative } \\
\text { (11) }\end{array}$ & $\begin{array}{l}\text { Thematic } \\
\text { analysis }\end{array}$ & Low \\
\hline Li, 2016 [61] & $\begin{array}{l}1 \text { January } \\
2000 \text { to } 21 \\
\text { February } \\
2015\end{array}$ & $\begin{array}{l}\text { Total: N = } 192434 \\
\text { PLHIV including } \\
\text { adults, children, } \\
\text { adolescents, } \\
\text { pregnant and } \\
\text { postpartum women, } \\
\text { and caregivers. }\end{array}$ & Adherence & $\begin{array}{l}\text { Botswana, Tanzania } \\
\text { and Uganda (1), } \\
\text { Peru (1), Ukraine (1), } \\
\text { Zambia (1), Rwanda } \\
\text { (1), Ethiopia (1), } \\
\text { Uganda (1), Nepal } \\
\text { (2), Cuba (1), } \\
\text { Southern Malawi (1), } \\
\text { Uganda and } \\
\text { Zimbabwe (1), China } \\
\text { (2), Tanzania (3), } \\
\text { South Africa (3) }\end{array}$ & $\begin{array}{l}\text { US (14), Netherlands } \\
\text { (1), Canada (1), } \\
\text { Australia (1), } \\
\text { Belgium and } \\
\text { Netherlands (1), } \\
\text { Switzerland (1) } \\
\end{array}$ & $\begin{array}{l}39 \text { studies: } \\
\text { Qualitative } \\
\text { (39) }\end{array}$ & $\begin{array}{l}\text { Thematic } \\
\text { analysis }\end{array}$ & Medium \\
\hline $\begin{array}{l}\text { Lytvyn, } 2017 \\
\text { [62] }\end{array}$ & $\begin{array}{l}1 \text { January } \\
2000 \text { to } 11 \\
\text { February } \\
2017\end{array}$ & $\begin{array}{l}N=1165 \text { : Women } \\
\text { considering } \\
\text { pregnancy (140), } \\
\text { pregnant women } \\
\text { (408), and } \\
\text { postpartum women } \\
\text { (602). Couples } \\
\text { desiring and/or } \\
\text { intending to have } \\
\text { children (15) also } \\
\text { included. }\end{array}$ & Adherence & $\begin{array}{l}\text { Puerto Rico (1), } \\
\text { Nigeria (1), Kenya } \\
\text { (2), Swaziland (2), } \\
\text { Malawi (2), India (1), } \\
\text { South Africa (1), } \\
\text { Zimbabwe (1), and }\end{array}$ & Australia (1), US (3) & $\begin{array}{l}15 \text { Studies: } \\
\text { Qualitative } \\
(15)\end{array}$ & $\begin{array}{l}\text { Meta- } \\
\text { ethnography }\end{array}$ & High \\
\hline $\begin{array}{l}\text { Merten, } 2010 \\
\text { [63] }\end{array}$ & $\begin{array}{l}2000 \text { to } \\
2008\end{array}$ & $\begin{array}{l}N=2044+ \\
\text { Community } \\
\text { members, policy } \\
\text { makers, PLHIV, } \\
\text { health workers, } \\
\text { female HIV+ } \\
\text { patients, healthcare } \\
\text { actors, In-school and } \\
\text { out-of-school youth, } \\
\text { patients who } \\
\text { attended the ARV }\end{array}$ & Adherence & $\begin{array}{l}\text { Uganda (6), Zambia } \\
\text { (5), South Africa (6), } \\
\text { Burkina Faso (1), } \\
\text { Malawi (2), Tanzania } \\
\text { (5), Botswana (2), } \\
\text { Kenya (1), Nigeria } \\
\text { (1), Ethiopia and } \\
\text { Uganda (1), Burkina } \\
\text { Faso, Cote d'Ivoire } \\
\text { and Mali (1), Nigeria, } \\
\text { Tanzania and }\end{array}$ & None & $\begin{array}{l}32 \text { studies: } \\
\text { Qualitative } \\
(32)\end{array}$ & $\begin{array}{l}\text { Meta- } \\
\text { ethnography }\end{array}$ & Low \\
\hline
\end{tabular}


Table 2 Table of included studies ( $N=33$ ) (Continued)

\begin{tabular}{|c|c|c|c|c|c|c|c|c|}
\hline \multirow{2}{*}{$\begin{array}{l}\text { First author, } \\
\text { year of } \\
\text { publication } \\
\text { [reference] }\end{array}$} & \multirow{2}{*}{$\begin{array}{l}\text { Search } \\
\text { dates }\end{array}$} & \multirow[t]{2}{*}{ Participants } & \multirow[t]{2}{*}{ Issue } & \multicolumn{2}{|l|}{ Context } & \multirow{2}{*}{$\begin{array}{l}\text { Types of } \\
\text { studies }\end{array}$} & \multirow{2}{*}{$\begin{array}{l}\text { Method of } \\
\text { synthesis }\end{array}$} & \multirow{2}{*}{$\begin{array}{l}\text { Overall } \\
\text { quality } \\
\text { of } \\
\text { review }\end{array}$} \\
\hline & & & & $\begin{array}{l}\text { Low- to middle- } \\
\text { income country }\end{array}$ & $\begin{array}{l}\text { High-income } \\
\text { country }\end{array}$ & & & \\
\hline & & $\begin{array}{l}\text { clinic, counsellors, } \\
\text { HIV+ patients on } \\
\text { ART for } 6 \text { months, } \\
\text { care givers, family } \\
\text { care givers, key in- } \\
\text { formants, HIV+ pa- } \\
\text { tients from IDP } \\
\text { camps, treatment } \\
\text { partners }\end{array}$ & & Uganda (1) & & & & \\
\hline Mey, 2016 [64] & $\begin{array}{l}\text { January } \\
2000 \text { to } 15 \\
\text { December } \\
2015\end{array}$ & $\begin{array}{l}\text { PLHIV, Men, women, } \\
\text { MSM, caregivers of } \\
\text { children who are } \\
\text { HIV positive, CAM } \\
\text { workers (traditional } \\
\text { healers/alternative } \\
\text { medicines) }\end{array}$ & $\begin{array}{l}\text { Linkage } \\
\text { Adherence } \\
\text { Retention }\end{array}$ & None & Australia (21) & $\begin{array}{l}35 \text { Studies: } \\
\text { Qualitative } \\
\text { (14), } \\
\text { Quantitative } \\
\text { (14), Mixed } \\
\text { Methods (6), } \\
\text { and Case } \\
\text { Report (1) }\end{array}$ & $\begin{array}{l}\text { Narrative } \\
\text { synthesis }\end{array}$ & Low \\
\hline $\begin{array}{l}\text { Mills, } 2006 \\
{[65]}\end{array}$ & $\begin{array}{l}\text { Up to June } \\
2005\end{array}$ & $\begin{array}{l}\text { PLHIV and caregiver } \\
\text { Total: } N=12902\end{array}$ & Adherence & $\begin{array}{l}12 \text { studies were } \\
\text { conducted in } \\
\text { developing } \\
\text { countries included } \\
\text { four from Brazil and } \\
\text { one each from } \\
\text { Uganda, Cote } \\
\text { d'lvoire, South } \\
\text { Africa, Malawi, } \\
\text { Botswana, Costa } \\
\text { Rica, Romania, and } \\
\text { China. }\end{array}$ & $\begin{array}{l}\text { US (56), Canada (3), } \\
\text { UK (3), Italy (2), } \\
\text { France (2), The } \\
\text { Netherlands (2), } \\
\text { Australia (1), } \\
\text { Belgium (1) and } \\
\text { Switzerland (1). The } \\
\text { studies conducted } \\
\text { in developing } \\
\text { countries included } \\
\text { Brazil (1) and } \\
\text { Botswana (1) Two } \\
\text { studies were multi- } \\
\text { national: (countries } \\
\text { not reported). }\end{array}$ & $\begin{array}{l}\text { 84 studies: } \\
\text { Qualitative } \\
\text { (37) and } \\
\text { Quantitative } \\
(47)\end{array}$ & $\begin{array}{l}\text { Content } \\
\text { analysis }\end{array}$ & High \\
\hline $\begin{array}{l}\text { Morales- } \\
\text { Aleman, } 2014 \\
{[66]}\end{array}$ & $\begin{array}{l}\text { Jan } 2002 \text { to } \\
\text { April } 2013\end{array}$ & $\begin{array}{l}N=121 \text { Hispanic } \\
\text { and Latino PLHIV }\end{array}$ & $\begin{array}{l}\text { Linkage } \\
\text { Adherence } \\
\text { Retention }\end{array}$ & None & USA (4) & $\begin{array}{l}3 \text { studies: } \\
\text { Qualitative } \\
\text { (3) and } \\
\text { Quantitative } \\
\text { (1) }\end{array}$ & $\begin{array}{l}\text { Thematic } \\
\text { analysis }\end{array}$ & Low \\
\hline $\begin{array}{l}\text { Omonaiye, } \\
2018 \text { [67] }\end{array}$ & $\begin{array}{l}\text { Up to } \\
\text { December } \\
2017\end{array}$ & $\begin{array}{l}\text { HIV-positive } \\
\text { pregnant women } \\
\text { (include number) }\end{array}$ & Adherence & $\begin{array}{l}\text { Kenya (3), Swaziland } \\
(1), \text { Uganda (2), } \\
\text { South Africa (1), } \\
\text { Cote d'voire (2), } \\
\text { Tanzania (1), Malawi } \\
\text { (4), Mozambique (1) }\end{array}$ & None & $\begin{array}{l}15 \text { Studies: } \\
\text { Qualitative } \\
\text { (9) and } \\
\text { Mixed } \\
\text { Methods (6) }\end{array}$ & $\begin{array}{l}\text { Thematic } \\
\text { content } \\
\text { analysis }\end{array}$ & Medium \\
\hline $\begin{array}{l}\text { Reisner, } 2009 \\
\text { [68] }\end{array}$ & $\begin{array}{l}1999 \text { to } \\
2008\end{array}$ & $\begin{array}{l}N=5179 \text { HIV- } \\
\text { positive youth and } \\
\text { adolescents and } \\
\text { pregnant } \\
\text { adolescents. }\end{array}$ & Adherence & None & US (14) & $\begin{array}{l}\text { 14 Studies: } \\
\text { Qualitative } \\
\text { (4), } \\
\text { Quantitative } \\
\text { (7) and } \\
\text { Mixed } \\
\text { Methods (3) }\end{array}$ & $\begin{array}{l}\text { Thematic } \\
\text { content } \\
\text { analysis }\end{array}$ & Low \\
\hline $\begin{array}{l}\text { Santer, } 2014 \\
{[69]}\end{array}$ & $\begin{array}{l}1996 \text { to } \\
2011\end{array}$ & $\begin{array}{l}N=96 \text { Caregivers of } \\
\text { children aged } 0-18 \\
\text { years }\end{array}$ & Adherence & None & $\begin{array}{l}\text { Belgium (1) and US } \\
\text { (2) }\end{array}$ & $\begin{array}{l}\text { 3 Studies: } \\
\text { Qualitative } \\
\text { (3) }\end{array}$ & $\begin{array}{l}\text { Thematic } \\
\text { analysis }\end{array}$ & Low \\
\hline $\begin{array}{l}\text { Vervoort, } \\
2007 \text { [70] }\end{array}$ & $\begin{array}{l}1996 \text { to } \\
2005\end{array}$ & $\begin{array}{l}N=1053 \text { Adult } \\
\text { PLHIV }\end{array}$ & Adherence & Not specified & Not specified & $\begin{array}{l}24 \text { studies } \\
\text { containing } \\
\text { qualitative } \\
\text { data. }\end{array}$ & $\begin{array}{l}\text { Thematic } \\
\text { content } \\
\text { analysis }\end{array}$ & Low \\
\hline $\begin{array}{l}\text { Vitalis, } 2013 \\
\text { [71] }\end{array}$ & $\begin{array}{l}\text { Up to July } \\
2011\end{array}$ & $\begin{array}{l}\text { HIV-positive } \\
\text { pregnant and } \\
\text { postpartum women } \\
\text { between the ages }\end{array}$ & Adherence & $\begin{array}{l}\text { Africa (7), Brazil (2) } \\
\text { and Puerto Rico (1) }\end{array}$ & $\begin{array}{l}\text { USA (8), and } \\
\text { Australia (1) }\end{array}$ & $\begin{array}{l}18 \text { studies: } \\
\text { Quantitative } \\
\text { (15) and } \\
\text { Qualitative }\end{array}$ & $\begin{array}{l}\text { Content } \\
\text { analysis }\end{array}$ & Low \\
\hline
\end{tabular}


Table 2 Table of included studies ( $N=33$ ) (Continued)

\begin{tabular}{|c|c|c|c|c|c|c|c|c|}
\hline \multirow{2}{*}{$\begin{array}{l}\text { First author, } \\
\text { year of } \\
\text { publication } \\
\text { [reference] }\end{array}$} & \multirow{2}{*}{$\begin{array}{l}\text { Search } \\
\text { dates }\end{array}$} & \multirow[t]{2}{*}{ Participants } & \multirow[t]{2}{*}{ Issue } & \multicolumn{2}{|l|}{ Context } & \multirow{2}{*}{$\begin{array}{l}\text { Types of } \\
\text { studies }\end{array}$} & \multirow{2}{*}{$\begin{array}{l}\text { Method of } \\
\text { synthesis }\end{array}$} & \multirow{2}{*}{$\begin{array}{l}\text { Overall } \\
\text { quality } \\
\text { of } \\
\text { review }\end{array}$} \\
\hline & & & & $\begin{array}{l}\text { Low- to middle- } \\
\text { income country }\end{array}$ & $\begin{array}{l}\text { High-income } \\
\text { country }\end{array}$ & & & \\
\hline & & $\begin{array}{l}\text { of } 12 \text { to } 58 \text { years } \\
\text { receiving ART. }\end{array}$ & & & & (3) & & \\
\hline $\begin{array}{l}\text { Wasti, } 2012 \\
\text { [72] }\end{array}$ & $\begin{array}{l}1996 \text { to } \\
2010\end{array}$ & $\begin{array}{l}N=4782 \text { Adult } \\
\text { PLHIV who have } \\
\text { been prescribed } \\
\text { ART. Quantitative } \\
\text { Studies } n=4372 ; \\
\text { qualitative studies } n \\
=152 \text { and mixed } \\
\text { methods studies } n \\
=258\end{array}$ & Adherence & $\begin{array}{l}\text { India (10), China (4), } \\
\text { Thailand (3), } \\
\text { Cambodia (1). }\end{array}$ & None & $\begin{array}{l}18 \text { studies: } \\
\text { Quantitative } \\
\text { (12), } \\
\text { Qualitative } \\
\text { (4) and } \\
\text { Mixed } \\
\text { Methods (2) }\end{array}$ & $\begin{array}{l}\text { Thematic } \\
\text { analysis }\end{array}$ & Low \\
\hline $\begin{array}{l}\text { Williams, } 2018 \\
\text { [73] }\end{array}$ & $\begin{array}{l}\text { January } \\
2005 \text { to } \\
\text { March } 2016\end{array}$ & $\begin{array}{l}\text { Adolescent ages 9- } \\
20 \text { years living with } \\
\text { HIV }\end{array}$ & $\begin{array}{l}\text { Linkage } \\
\text { Adherence } \\
\text { Retention }\end{array}$ & $\begin{array}{l}\text { Zimbabwe (2), } \\
\text { South Africa (3), } \\
\text { Kenya (3), Botswana } \\
\text { (1), Zambia (3), } \\
\text { Tanzania (1), } \\
\text { Uganda (1), Uganda } \\
\text { and Zimbabwe (1), } \\
\text { Tanzania (2), and } \\
\text { Botswana and } \\
\text { Tanzania (1) }\end{array}$ & None & $\begin{array}{l}18 \text { studies: } \\
\text { Qualitative } \\
\text { (18) }\end{array}$ & $\begin{array}{l}\text { Meta- } \\
\text { ethnography }\end{array}$ & Low \\
\hline
\end{tabular}

PLHIV doubted their ability to adhere and commit to lifelong treatment and care. Themes for the facilitators of linkage to ART on the individual level included physical health (6 findings) and barriers regarding physical health (8 findings). In the context of participants' psychological distress, some reviews found that participants could no longer ignore the physical symptoms of the disease or their declining health, while others found that although they tested positive for HIV, they were asymptomatic, and therefore delayed care. The desire to care for family, protect unborn children from the transmission of HIV, as well as the desire for future marriage and children facilitated children's linkage.

On the interpersonal level, relationships in the household emerged as an important theme, both as barriers (7 findings for adults and 1 finding for children) and facilitators (3 findings for adults and 1 finding for children). PLHIV reported conflicts in the household, threats of domestic violence and abandonment, and the lack of autonomy for women, as barriers to linkage to ART. In contrast, supportive partners and families with mutuality-fostering relationships involving empathy facilitated linkage.

On the community level, the main barrier expressed was stigma and discrimination (4 findings), which is linked to community narratives around masculinity, HIV as witchcraft, and hospitals as places of death. Children reported unsupportive teachers (1 finding) as a barrier to linkage. Facilitators reported included peer support and support groups (6 findings) which served as a proxy for family support when it was lacking. One finding for adults included community beliefs and practices as a barrier with negative beliefs about ART, detrimental gender norms, and a preference for traditional healers and medicines.

At the institutional level, barriers such as stigma experienced at health care facilities (7 findings), service delivery (24 findings), which includes overcrowding, long queues, high staff turnovers, inconvenient client times, poor resources and participants' experiences with limited medication availability as well as their experiences of HIV testing were reported. Four findings were related to barriers of institutional models of care. PLHIV identified gaps in the ART cascade referral process, particularly for women who test positive during their antenatal care (ANC) and are not followed-up postpartum, as well as lack of integrated services. PLHIV perceived health care models such as home visiting, as a barrier, as it might contribute to involuntary disclosure. There were fourteen findings for facilitators in the theme models of care, including offering population-specific services for adolescents, the integration of HIV care within ANC, offering mental health assessments and providing multilevel, multi-pronged approaches to care. The facilitators for the theme service delivery ( 2 findings) included PLHIV having positive experiences of HIV testing and encountering a clinic staff member who welcomed people into the clinic. Counselling practices and principles (6 findings) that respected the place of traditional medicine, incorporated the traditional beliefs of people, 


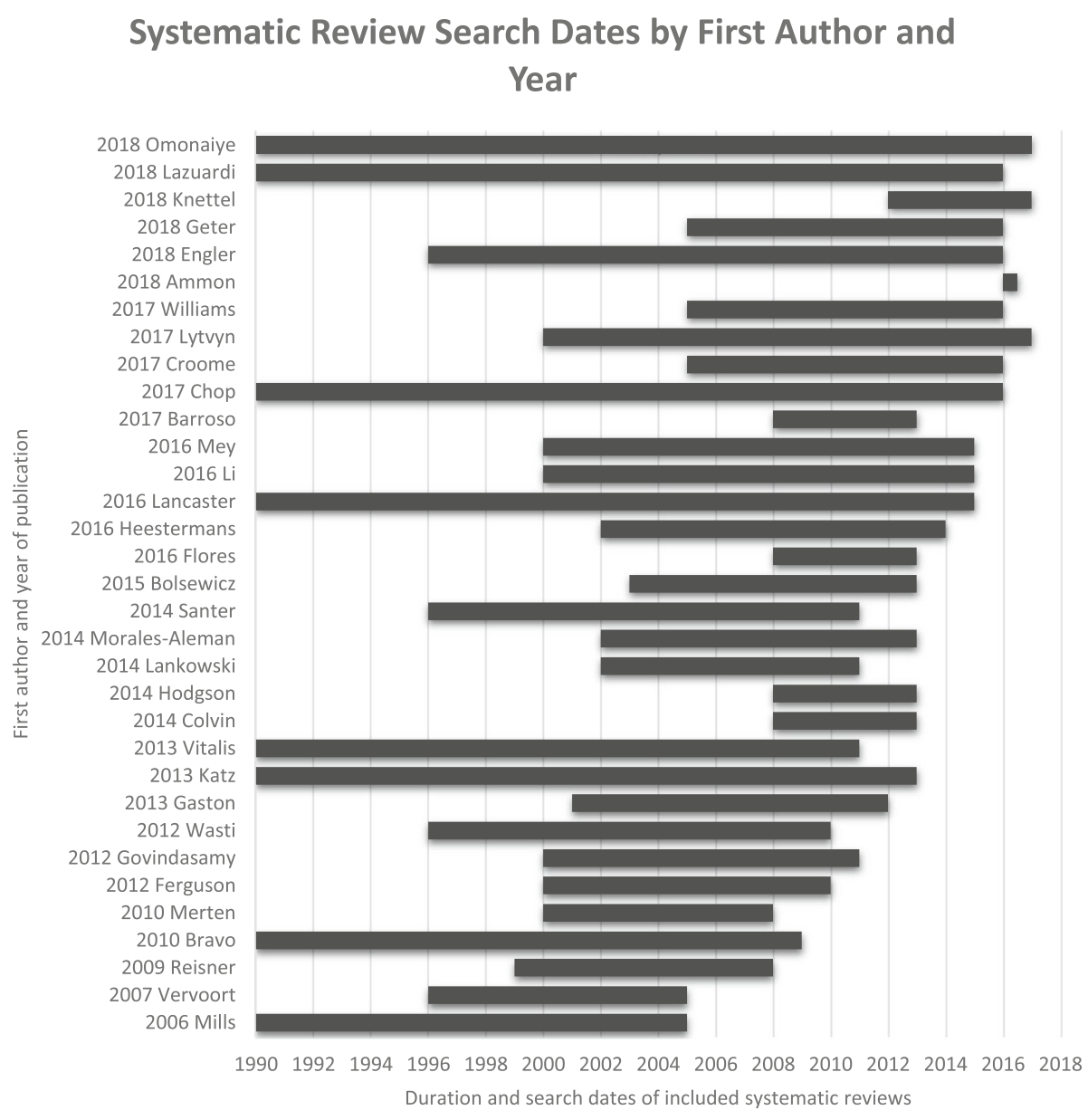

Fig. 5 Overlap between search dates of included systematic reviews ( $N=33$ )

and that provided in-depth counselling before and after HIV testing were reported as facilitators on the institutional level. Children reported lack of privacy experiences at the clinic, the physical environment at the clinic and high staff turnover as barriers to linkage (1 finding).

On the structural level, reported barriers included the financial cost of care (2 findings), healthcare policies (3 findings), income and food security (4 findings), transport and distance to the clinic (4 findings) and one finding for living conditions and context. Facilitators included income and food security (1 finding), and transport and distance to clinic (2 findings), which includes having an escort to the clinic.

Only one high-quality review [65] was found that addressed linkage to care for HIV-positive adults. One theme was included from the high-quality review, namely, medication as a reminder of HIV status, within the individual level of the framework.

\section{Barriers and facilitators to adherence to ART}

Findings on barriers and facilitators to adherence to ART, and reviews reporting on these are summarised in
Additional file 11. For children, one high-quality review [65], one medium-quality review [41] and two lowquality reviews $[68,73]$ contributed to the aggregation of findings. For adults, two high-quality reviews [62, 65], four medium-quality reviews $[43,55,61,67]$ and twenty low-quality reviews $[44,47,48,50-56,58-68,70-73$ ] contributed to the findings for adherence to ART.

On the individual level, emerging themes related to barriers to adherence were linked to medication (19 findings), sociodemographic factors (18 findings) and fears (12 findings). Reviews reported medication characteristics, negative side effects, pill burden and regimen, travelling away from home and lack of privacy as barriers, and the use of reminders, simpler medication regimens as facilitators, within the medication theme. Several reviews synthesised findings on the self-reported sociodemographic characteristics such as levels of education, age and gender. In some cases, a woman's positive HIV status was considered a result of her husband's infidelity and reduced the risk of stigmatisation when disclosing her status. In other cases when women were seen taking their medication they were stigmatised as 


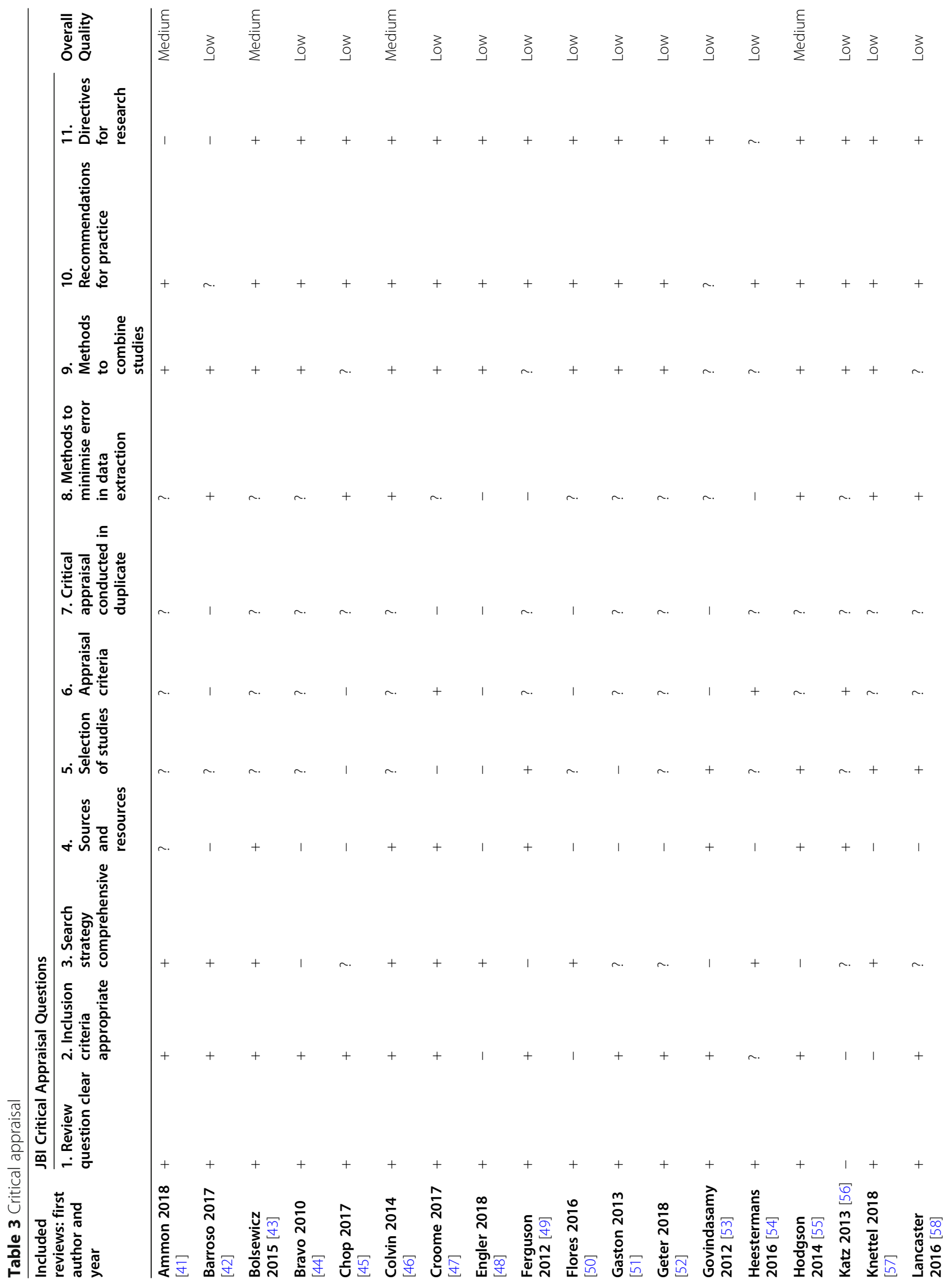




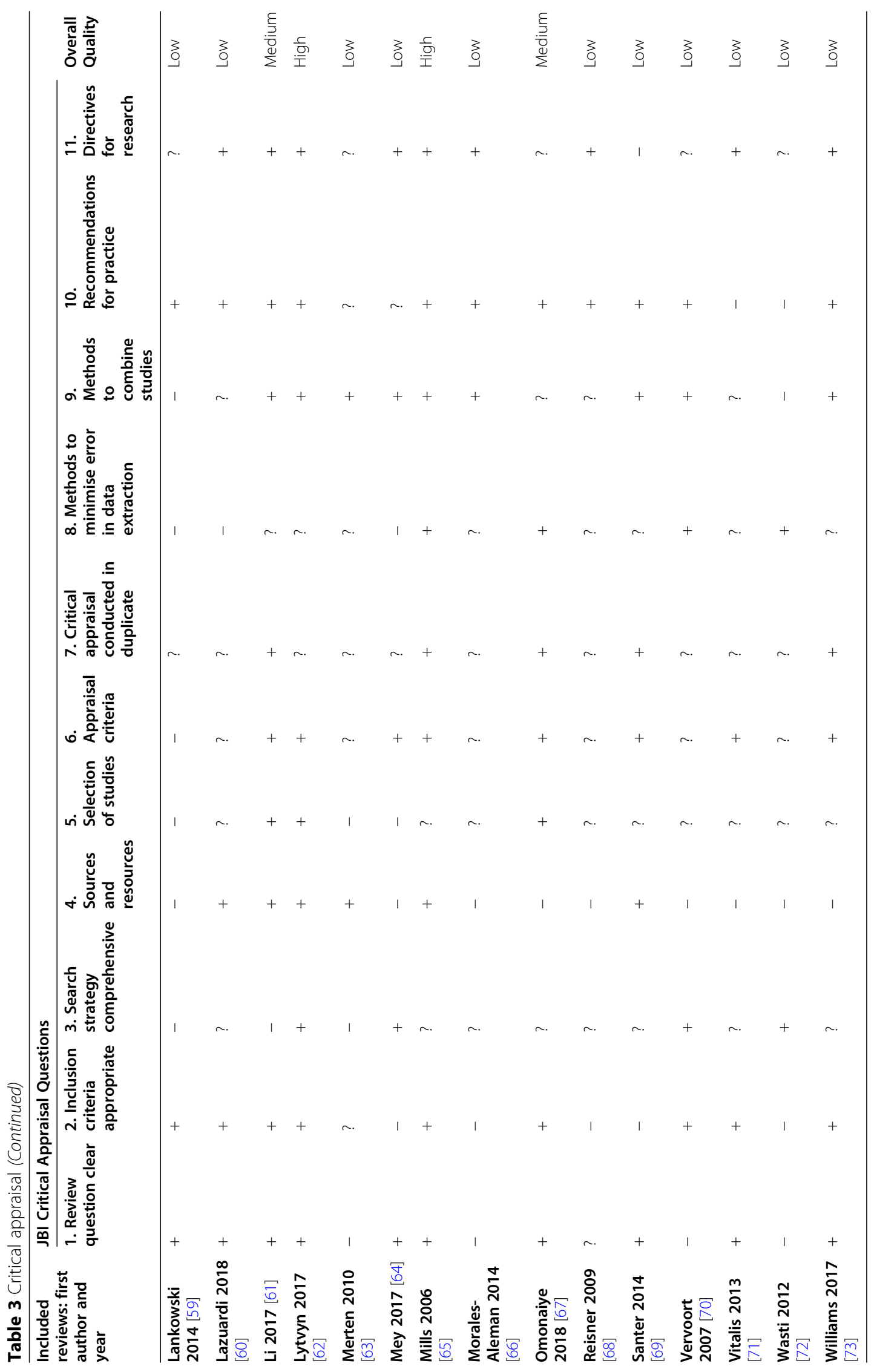


hypersexual and were discriminated against. Other reported barriers were grouped under the themes psychological distress and emotions (15 findings) and fears (12 findings). Fears were related to the medication toxicities, side effects, unintentional disclosure, that the treatment would harm a pregnant woman's unborn child and the fears that ART leads to impotency, infertility, and the impossibility of sexual activity.

The theme of coping strategies (12 findings) and desires (7 findings) were identified as facilitators to mitigate fears, anticipated stigma and negative side effects of ART. Coping strategies included being aware of personal strengths and weakness, learning to manage the HIV diagnosis and interpreting physical signs of the body, drinking liquids, resting and adopting a resilient and positive attitude. People desired to be healthy to care for their families and to maintain their appearance to keep their status a secret. Knowledge and understanding was identified both as a barrier (6 findings), such as receiving conflicting messages from community members, providers, peers and the media; and as a facilitator (7 findings) such as, understanding the need for compliance.

On the interpersonal level, peoples' relationships within the household emerged as a barrier (15 findings) and facilitator (7 findings) to adherence. Family involvement and emotional, material, and social support were important factors to PLHIV. Other barriers such as punishment for lack of adherence for children, negative family reactions to disclosure, enacted stigma by family members and lack of autonomy in relationships made it difficult for people to adhere.

Community level barriers were related to community beliefs and practices (6 findings) such as strong negative community beliefs about HIV and bypassing of clinics and hospitals for traditional healers. Peers and support groups (6 findings) played a mitigating role and helped participants adjust to their new daily routine. Financial and emotional support also facilitated adherence.

The two emergent themes identified at the institutional level of the framework were service delivery (27 findings), which was reported as a barrier; and models of care (15 findings), which was reported as a facilitator. People who may have had the intention of adhering to ART were discouraged by the difficulties of making a scheduled appointment and the long waiting times at the clinic when they did seek care. Negative experiences at the clinic when collecting the refills of medication included the lack of privacy, overcrowding and stigma experienced within the clinic by other patients, community members and staff. People reported spending up to a day waiting to see a health care worker and were presented with additional barriers such as drug stock-outs or limits on the amount of medication that could be dispensed at a time. Models of care, such as integrated mental health care, integrated antenatal care (ANC) and HIV care, and specialised services for adolescents, with highly skilled and trained healthcare workers,

The structural-level themes identified for barriers to ART adherence included the financial cost of ART, healthcare policies, and income and food security, each of which had three findings. PLHIV reported that food insecurity and no access to liquids prevented them from taking their medication. PLHIV felt discouraged by their lack of understanding of healthcare policies and some reported the barrier of access laws at health care facilities that sent patients to their birthplace to seek care. Policies directed at specific populations with criminalising threats for transgender persons, commercial sex workers, drug users and deportation threats for immigrants were reported as barriers. Even with the advent of free ART, the indirect cost of ART is still high in low-income settings with participants expressing the challenge of travelling to clinics in rural areas, the affordability of safe, reliable transport and the indirect cost of childcare when visiting the clinic in order to collect medications. Facilitators at the structural level included financial relief for care (5 findings) and income and food security ( 2 findings), which included the provision of grants for food supplementation and travel reimbursement.

One high-quality review [65] found that children reported their daily routines and lifestyle, desires to have their lives pre-ART normalised, fears of stigma, fears of the related effects of ART as well as actual negative effects experienced, non-acceptance of HIV status, conflicting messages regarding ART, forgetting or misplacing medication, medication characteristics, pill burden, feeling better, unsupportive family relationships and social isolation as barriers to their medication adherence. No facilitators of adherence to ART for children were reported in the high-quality review. Review findings from two high-quality reviews found that adults reported their beliefs about ART, coping strategies, daily routines, desires, fears, HIV acceptance and nonacceptance, knowledge and understanding of ART, medication factors, physical health, psychological distress, age and competing life interests on the individual level. Relationships in the household on the interpersonal level of the framework; peer and social support groups on the community level; perceptions and engagements of health care workers, integrated models of care, male only services, health care workers' recommendations, service delivery, financial costs and health care policies on the institutional level; and food insecurity, housing and income as structural factors were self- 
reported by people as barriers and facilitators of adherence to ART.

\section{Barriers and facilitators to retention in care}

Ten reviews contributed to the findings for retention in care, and the barriers and facilitators as reported in the reviews, by country income level and quality rating, are presented in Additional file 12. Five low-quality reviews $[50,52,58,66,73]$ reported on the barriers and facilitators of retention in care for children. Findings for adults were found in seven low-quality reviews $[42,50,52,56-$ $58,66]$ and two medium-quality reviews $[46,55]$. No medium- or high-quality reviews were found for children and no high-quality studies were found for the PLHIV self-reported barriers and facilitators of retention in care.

The prominent themes on the individual level were the barriers of sociodemographic factors (5 findings), such as issues around gender, and concerns about not have registered identification documents to access care due to either immigrant status or being transgender; the themes of fears (4 findings); and psychological distress and emotional reactions (7 findings). PLHIV reported experiencing mental fatigue from being retained in care and experiencing psychological suffering as an adult with HIV, which included feeling angry, feeling like they have lost control of their lives and feelings of depression and hopelessness.

On the interpersonal level, emergent themes included the barriers ( 5 findings) and facilitators (3 findings) of disclosure. Disclosure was reported as a barrier either in cases when participants chose not to disclose, and this resulted in sporadic care within health care systems, or due to post-disclosure stigma. Family members who were supportive and relationships in the household (6 findings) were reported as facilitators of retention in care. Partners who were emotionally supportive and encouraged healthy living were considered facilitators of retention in care. In other instances, partners who were not involved in care were considered as barriers, with reviews reporting that women did not have decisionmaking power in some contexts, and this denied them the opportunity to seek care.

The community level had two emergent themes with one finding each. These included the theme of community beliefs and practices and the theme of peers and support groups, which was also reported as facilitators with six key findings. Facilitator findings included having a treatment companion, identifying a confidante, attending support groups and enlisting the help of supervisors and teachers to facilitate retention in care.

For retention in care, many themes that related to the institutional level were reported. Themes included service delivery (20 findings) and models of care (17 findings), followed by stigma in health care and engagement
(7 findings) and engagement with health care workers (6 findings). Service delivery barriers included long waiting times and subsequent short consultations with health care workers, drug and test stock outs, lack of privacy, laboratory challenges, negative experiences of testing for HIV, the physical clinic environment and the failure of the health care facility to keep up with rapidly changing treatment protocols. PLHIV reported same-day appointments between services offerings at the clinic, their experiences of HIV testing and the provision of disability accommodations to be facilitators of service delivery ( 5 findings). The facilitators of models of care (9 findings) included integrated care to reduce patient burden, the treatment of depression and anxiety related to diagnosis, offering male-friendly services without needing to access care through partners' ANC services and home visiting or mobile care units.

Structural-level barriers were emergent for health care policies (4 findings), financial costs of care (2 findings), transport and distance to clinic (2 findings), and one finding each for income and food security, and living condition and context. PLHIV reported the cost of attending care even while ART was universally free and accessible as a barrier to engaging in care. Indirect costs included the loss of wages when attending the clinic, transportation costs, childcare costs and the possible loss of grants due to their HIV-positive diagnosis. Only one facilitator of having a higher income was reported at the structural level.

No high-quality reviews were found for self-reported barriers and facilitators of retention in care for children or adults.

\section{Synthesis of findings \\ Identifying themes and subthemes}

We reclassified the third-order concepts into 45 fourthorder themes within the five levels of the Kaufman [23] HIV behaviour change model and summarised the themes of included reviews linked to the outcomes (Additional file 13). For the individual level, we found 19 themes; for the interpersonal level, five themes; for the community level, six themes; for the institutional level, eight themes; and for the structural level, we found six themes (Fig. 6).

\section{What are the knowledge gaps in the available review-level evidence about linkage to care?}

Review-level evidence (Additional file 9) on the barriers and facilitators to linkage to ART for children is sparse, with a single review reporting on children and adolescents in low- and middle-income countries. A lowquality review identified the main barriers for children in both low- and middle-income countries were categorised into the interpersonal and institutional level of the HIV 


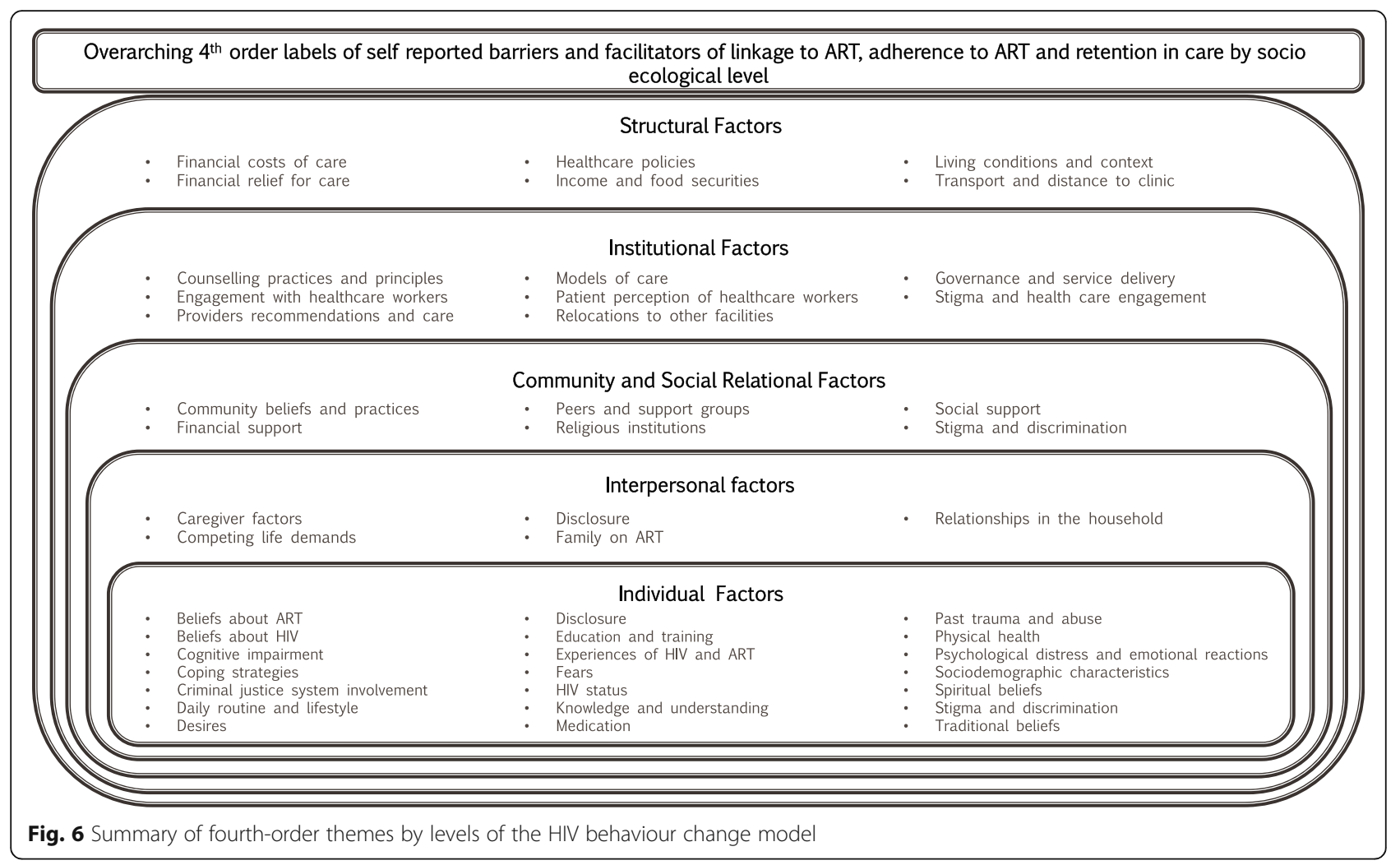

behaviour change model. Review-level evidence is lacking for the psychological distress and emotional reactions experienced by children and adults when learning about their positive status and possible mode of transmission, whether through unprotected sex or vertical transmission. Furthermore, the mental health of PLHIV as they engage in the continuum of care is underrepresented in the literature. Review-level evidence of the facilitators of linkage are under-reported, as are children's perceptions of and engagement with health care workers. No high-quality reviews reporting on children's self-reported barriers and facilitators to linkage were found. For adults, much of the review-level evidence has synthesised the barriers of linkage to ART rather than the facilitators. With only one high-quality review, there is a need for more high-quality reviews on the facilitating effect of community beliefs and practices, internal beliefs and the role of peers and support groups for linkage to treatment for ART. Additionally, there is lack of evidence on the coping strategies employed by children and adults to facilitate linkage. There is a need for highquality evidence on environmental factors and the social determinants of health on people's linkage to ART.

\section{What are the knowledge gaps in the available review-level evidence about adherence to treatment?}

There is minimal review-level evidence for children when compared to the existing evidence of adherence to
ART in adult populations (Additional file 10). We found only one high-quality review for children and two highquality reviews for adults. However, when comparing the methodological quality of reviews, there is a large body of low-quality reviews, with most reviews being conducted on populations from low-income countries. Individual beliefs, desires, coping strategies and fears are addressed in the literature for adults but not adequately for children. No review-level evidence was available on the relationships within the household in low- and middle-income countries for children. There is a large quantity of evidence on the barriers of the characteristics of the medication, the side effects, the psychological distress and emotional reactions and the effects of service delivery but a gap exists in the evidence of the facilitators that can mitigate these barriers in adults. Evidence in adults is lacking on the role of environmental factors, personal beliefs, cultural practices and traditional community beliefs on adherence to ART. Further exploration on the self-reported experiences of psychological distress and engagement with peers in the context of stigma is needed.

\section{What are the knowledge gaps in the available review-level evidence about retention in care?}

The existing evidence for retention in care is sparse (Additional file 11), and no high-quality reviews were found. The focus of synthesis from the included studies 
has primarily been conducted in adult populations in low- and middle-income countries. Five low-quality reviews and one medium-quality review addressed the outcome of retention in care among children and no highquality reviews were found. In children, caregivers are seen as the primary caregivers and gatekeepers for management of a positive HIV diagnosis, but this is not displayed in the existing evidence, as there is a lack of research that is available. Additionally, the role of caregivers in adult populations was not available in the included reviews. In low- and middle-income countries, complexities regarding the co-existence of traditional medicine and scientific-based medicine are briefly mentioned, but not explored, in review-level evidence. Adult population self-reports of family and social relationships are synthesised in low- and middle-income countries as well as high-income countries; however, there is no review-level evidence for children. There is a need for more review-level evidence on the impact of the environment and structural community on retention in care. No high-quality reviews were found for PLHIV reporting barriers and facilitators to retention in care.

\section{Lines of action and recommendations}

Several of the individual, interpersonal, health system and structural-level factors identified in this overview are well known and have been identified in previous literature $[35,75]$. We identified key areas and population groups which were under-represented in the review literature and where syntheses of primary research may be needed to understand these factors better.

First, individual-level factors such as peoples' fears, psychological distress, and beliefs about ART, and conflicting beliefs between cultural practices and medicine, were frequently reported; there was however little to no emphasis in qualitative reviews on the facilitators to mitigate these barriers in children or adult populations.

Second, interpersonal factors such as the dynamics of relationships within the household, and the fears, anxieties, experiences of disclosure and support from peers and support groups were identified both as facilitators and barriers to care; however, details about the complex relationship of these factors needs further exploration.

Third, models of care and service delivery practices adopted by health care centres were prominent themes: with long waiting times, the risk of unintentional disclosure and the negative treatment by health care workers reported as barriers. Skilled health care workers, targeted services for men and youth, and integration of care as facilitating care were identified. Further exploration of not only the healthcare workers, but also the clinic facility, both as a physical space and beliefs about the clinic, is needed.
Fourth, structural-level themes included transportation to the clinic, poverty and low-income levels linked to the lack of food. Emerging issues related to the sensorial experience of adherence, the physical environment and community structure has yet to be explored in qualitative systematic reviews related to HIV.

Fifth, although there is progress to achieving the 90-90-90 targets, this study identified many challenges experienced by PLHIV and the themes of selfreported barriers provide a rich overview of the interwoven complexities of the lives of PLHIV. Recent literature linking barriers and facilitators of linkage, adherence and retention in care to priority solutions propose differentiated service delivery, client-centred care, safe spaces for HIV treatment and care, adolescent friendly services, family centred services and advocacy for human rights, among others, to best serve the needs of PLHIV and reduce the burden on the health care systems. Investigation and scale up of interventions for PLHIV to promote their adherence must be intensified and barriers and facilitators even beyond the realm of interventions proposed including reminder devices, pill box organisers, reduced toxicity, mental health screening and fixed dosed combinations must be explored.

Sixth, this overview identifies the need for qualitative exploration of the agency of the material environment encountered by PLHIV, not included in existing reviews or in intervention research and will make valuable contributions to the body of knowledge. The exploration of agency goes beyond the identification and description of themes to exploring the understanding of the meaning ascribed to materials such as agency of the human body of PLHIV, the agency of matter such as money, transport vehicle, clinic environments, food, community structural factors and living spaces, context and agency of medications.

Lastly, this study identified the need for more highquality review-level evidence for key populations, specifically, children and youth with perinatal HIV infections, as they were underrepresented in the data. Most included reviews were assessed as having low quality. Future qualitative systematic reviews in the field of HIV linkage, adherence and retention in care should follow a rigorous approach to minimise the risk of bias.

\section{Discussion}

This study described the innovation and first application of the mega-aggregative framework synthesis approach to explore self-reported barriers and facilitators of care in PLHIV as reported in existing qualitative systematic reviews. The purpose of mega-aggregation framework synthesis is to provide an overview of review-level findings to produce lines of action which leads to 
recommendations. As the number of qualitative reviews increases exponentially $[18,19]$ and the risk of review duplication in HIV research is high, we considered it valuable to appraise and synthesise the existing evidence. This novel application was conducted on real reviewlevel data which identified 33 systematic reviews from low-, middle- and high-income countries, reporting on linkage to ART, adherence to ART and retention in HIV care, for all populations, across all settings, from 1990 up to July 2018. The protocol was predefined and the overview conducted and reported in a transparent way, with all evidence, data extraction, critical appraisal, justifications for decisions, clear decision guides and data presented in additional files alongside the manuscript. The search was comprehensive, and data extraction, screening and critical appraisal was conducted independently by two authors.

Mega-aggregation framework synthesis for qualitative reviews has been shown to be a reliable and efficient method of synthesis when aiming to identify the quality and scope of available evidence. Researchers are encouraged to use and build on the method as overviews become more prominent in qualitative systematic review literature. Our overview found that the main selfreported barriers and facilitators to linkage, adherence and retention such as psychosocial characteristics of PLHIV such as desires, fears, experiences of HIV and ART, coping strategies and mental health intersected with other factors on the interpersonal, community, institutional and structural level. PLHIV identified stigma and lack of social support, alongside health care services that were not sensitive to their individual needs as barriers to adherence and retention in care. In low-income countries, structural, community and institutional factors were reported frequently, and in high-income countries, individual-level barriers such as fear of medication side effects were more apparent. Although the overall quality of the reviews was low, the findings of this overview provide sufficient evidence to assist with the identification of knowledge gaps in the literature and clear lines of action for research, policy and practice. To understand the experiences of PLHIV when considering committing to lifelong treatment, the potential postdisclosure life changes and the side effects, juxtaposed with their beliefs about medication and diseases, it is important to consider all the factors on the individual, interpersonal, community, institutional and structural factors that influence their decision-making and actions. Practitioners working in health care cannot treat patients only for their diagnosis of HIV but must provide holistic care and be aware of the barriers and facilitators that these patients may be experiencing, from the patients' perspectives. Themes that were found varied across stages of the HIV cascade, and the reasons why PLHIV chose to adhere could be different to the reasons why they chose to engage in care or attend the clinic. The experiences of one patient may not be the experience of another and should be dealt with a case-by case manner within the contextual circumstance of the patient. The healthcare policies have been reported as not easily understood and methods of dissemination and education need to be considered for information uptake in communities. Exploration into the agency of the material spaces occupied and material items in the spaces of PLHIV will provide further understanding on the complex interplay of barriers and facilitators, especially in low- and middle-income countries. Community engagement workers can use their platforms to create open dialogues about HIV, the negative beliefs and stigmas, the dissemination of information on services that are provided in the communities and to promote the positive effects of staying adherent to ART for people infected with HIV. The findings of this overview provide researchers and practitioners with a broad overview of the existing evidence and are useful in the development of new research questions to respond to evidence gaps identified. It is known that the factors associated with linkage, adherence and retention in care do not occur in isolation but are in fact complex and tightly interwoven [43].

We first considered developing this method of synthesis after searching for an appropriate way to synthesise a large amount of qualitative systemic reviews. We wanted to know what was available, what was the quality of the evidence, and what new research questions should we invest our time and resources into. In the context of the pragmatic stance and the anticipated large number of existing systematic reviews, a predetermined theoretical framework [23] with broad categories was selected to guide the aggregation and synthesis within this overview, which built on the steps in methods development for conducting overviews [24], QES [25, 26], systematic review synthesis $[19,27,28]$, meta-aggregation $[9,20,21]$ and framework synthesis $[29,30]$. While conducting this overview, a worked example of mega-ethnography [8] was published. However, there was and there currently is no specific guidance on conducting a pragmatic synthesis in an overview of qualitative review-level evidence. We initially mapped out broad steps aligned to conventional systematic review methods with deviations in the analysis. As we worked through and reflected on the synthesis, we found it critical to identify the theoretical framework that had broad categories within which we could extract data at the outset of the protocol, and to produce lines of action and recommendations at the end of synthesis. We then refined the method into 8 steps which we illustrated in this manuscript. The steps include the following: (1) identify a clearly defined review 
question and objectives, (2) identify a theoretical framework or model, (3) decide on criteria for considering reviews for inclusion, (4) conduct searching and screening, (5) conduct quality appraisal of the included studies (although some may prefer not too), (6) data extraction and categorisation, (7) present and synthesise the findings, and (8) transparent reporting.

Before embarking on new systematic reviews, it is important for authors and review teams to take stock of existing evidence and minimise the risk of creating research waste. This overview highlights the need for more qualitative review-level evidence, with high methodological quality, that explores the complex relationships between structural factors and adherence, between sociodemographic factors such as community violence and retention, and the experiences of growing up with HIV in low- and middle-income countries, specifically in the populations of youth, women and key populations.

\section{Strengths and limitations of the mega-aggregative framework approach}

Through our worked application, we identified benefits of the mega-aggregative framework approach. First, the pragmatic philosophical underpinning promoted the aggregation of findings and a clear identification of where research is lacking, the quality of the research and the location of research across socio-economic statuses of countries. Some overviews only provide a synthesis without clear directives for policy and practice, and authors may see this as optional-leaving the reader to deduce or draw their own conclusions on the way forward. Using the mega-aggregative framework approach, the lines of action provide researchers and funders with clear guidance on where to direct funding and resources or to develop new research questions. It also provides practitioners with a comprehensive selection of the available evidence and the appraised quality of the evidence. A second benefit is that a mega-aggregation framework synthesis can be conducted in a relatively short amount of time. Other methods of synthesis can be iterative as review authors make sense of their own subjective interpretations. Using a framework with broad categories allows for linear working and promotes the comparing of findings between review authors. Using this method, the number of findings is counted and reported, with research gaps and recommendations for policy and practice being apparent immediately. Concrete time is then spent of the lines of action. This approach is feasible and can be especially attractive to novice researchers. A third benefit is the attention to the methodological quality of included systematic reviews; however, the benefits of the method proposed are not without its challenges or limitations. While there is ongoing debate [64, 65] about when, within which paradigmatic stance, and whether to conduct critical appraisal of included studies within a qualitative evidence synthesis, we included methodological appraisal as a step in the mega-aggregative approach. We found very few highquality reviews examining self-reported barriers and facilitators to linkage, adherence to ART and retention in care for children and adults. In the results, we presented an aggregation of findings and the provided a separate paragraph detailing the findings of the high-quality included reviews. This does not imply that the primary studies included in the reviews are of low quality, as we only assessed the methodological quality of the systematic reviews. We used guidance from recent methodological papers $[25,26,76-78]$ in the field of critical appraisal to revise the JBI-SR Checklist [78] for our overview and to determine our decision rules for overall quality of the reviews. The appraisal of the reviews challenged us as authors to think reflexively about the domains that influenced the quality of the reviews and the items on the appraisal tool that explored these domains. As with overviews of qualitative systematic reviews, the area for method development in critical appraisals of QES is understudied and tools for critical appraisal of QES are still under development. Using the mega-aggregative framework approach can limit the author team in their creativity or conceptual modelling of new theories. The mega-aggregative framework approach uses an existing framework and pragmatic approach that can make the analysis process mechanical and sometimes force the data into predefined categories. When we refined the steps of the method, we included selection of the framework as a critical step as much of the synthesis is defined by it. Using a framework with broad categories is preferable. Additionally, we experienced challenges related to the definitions of the outcomes of interest, namely, linkage, adherence and retention in care, in the included qualitative reviews, even though these were pre-specified in the protocol. In quantitative research, measurements are used to determine adherence, whereas qualitative data may be broader. We discussed these in the author team and decided to use overarching definitions and broad categorisation. It is recommended that future qualitative research interrogate the conceptual definitions of linkage, adherence and retention in care for PLHIV. We were challenged by our own assumptions that the pragmatic aggregative nature of the proposed synthesis would be easily applied to sub-group analysis. We intended to explore the data by subgroups for children $0-13$ years, young people and adolescents, perinatally infected adolescents and adult subgroup populations, but due to the different reporting methods and study designs, we were unable to provide a detailed synthesis of themes for these subgroups. We were also unable to group specific themes to specific population groups or contexts. However, we grouped children and youth as up to 24 years and adults as our second 
population group. Furthermore, we found a high proportion of reviews to be conducted in low- and middleincome countries. We conducted the aggregation for all studies and included evidence annexes indicating the socio-economic country grouping as low, middle, or high for each reference. Lastly, the synthesis of the findings was limited to the included review authors' interpretation of the data and we included the primary study data as per the review authors' description. It does become challenging for research synthesis as the production of QES is increasing and the risk of sampling and re-interpretation of the same primary studies for different outcomes and objectives through different paradigmatic lenses. We extracted themes from the reviews as per the authors' interpretation. However, using guidance from metaaggregation [9, 22], we only included themes that were considered unequivocal, meaning the themes were supported with evidence in the included review. We provided transparent decision-making notes and evidence annexes to this manuscript to contribute to the trustworthiness of the findings of this overview.

\section{Conclusion}

This paper describes the development and illustrates the first application of mega-aggregation framework synthesis for QES. The use of overviews to provide a synthesis of the existing review-level evidence is expected to increase as the pool of systematic reviews continues to grow. As with all research designs dependent on the research question and available resources, the aims and scope of QES-at review and overview level-are varied. Mega-aggregative framework synthesis is feasible and easy to implement and follows a linear process. Author teams interested in timeous and pragmatic ways to go beyond scoping reviews or evidence gap maps, into creating synthesised statements with practical lines of action and recommendations, are encouraged to experiment and utilise the illustrated innovative method of mega-aggregative framework synthesis for use in overviews of qualitative systematic reviews. Further evaluation and development of this method is needed to test its utility with different types of data and in comparison, to different approaches in qualitative synthesis.

\section{Supplementary Information}

The online version contains supplementary material available at https://doi. org/10.1186/s13643-021-01582-z.

Additional file 1. Search Strategies for electronic databases

Additional file 2. Revised decision rules for JBI-SR-Checklist

Additional file 3. Data extraction form

Additional file 4. Description and application of mega-aggregation

framework synthesis
Additional file 5:. PRISMA Extension for Scoping Reviews (PRISMA-SCR): Checklist

Additional file 6. Differences between protocol and manuscript Additional file 7. Tables of excluded studies, ongoing studies, and protocols $(N=45)$

Additional file 8. Individual summaries and critical appraisal reasons

Additional file 9. Overlap of included primary studies $(n=826)$

Additional file 10: Summary of review level evidence: Linkage to ART Additional file 11. Summary of review level evidence: Adherence to ART

Additional file 12. Summary of review level evidence: Retention in care Additional file 13. Summary of themes and included reviews linked to outcomes

\section{Abbreviations}

AIDS: Acquired immunodeficiency syndrome; ART: Antiretroviral therapy; HAART: Highly active antiretroviral therapies; HIV: Human immunodeficiency virus; PLHIV: People living with HIV; IDU: Intravenous drug user; JBI: Joanna Briggs Institute; JBI-SR Checklist: Joanna Briggs Systematic Review Checklist; MSM: Men who have sex with men; PREP: Pre-exposure prophylaxis; PRISMAScR: PRISMA Extension for Scoping Reviews Checklist

\section{Acknowledgements}

The authors would like to thank Vittoria Lutje and Anel Schoonees for conducting the searches. The authors would also like to acknowledge Taryn Young and Karin Hannes for their mentorship through the review process. We would like to acknowledge the authors that responded to our queries for additional information regarding their publications.

\section{Authors' contributions}

$L H, A R$ and EIW conceptualised the research question and LH wrote the protocol. AR and EIW reviewed and approved the protocol. LH and AR screened the titles and abstracts of studies resulting from the search as well as the full text included studies. LH and AR extracted data and critically appraised all included studies. IEW helped to resolve discrepancies for screening and data extraction. LH and AR conducted the mega-aggregation. LH wrote the final manuscript. AR and IEW read, reviewed and provided critical input into the final manuscript. All authors approved of the final manuscript.

\section{Authors' information}

$\mathrm{LH}$ is a practising research psychologist (M Psych) and holds a masters in Clinical Epidemiology. She is currently a PhD researcher and teaches qualitative evidence syntheses. She used her expertise in qualitative research to lead the conceptualisation of the protocol, the qualitative analysis of extracted data and the write up of this paper. IEW is an HIV specialist who assisted with the selection of relevant reviews, conceptualisation of the protocol, extraction and appraisal of the data, and writing the manuscript. $A R$ is an experienced systematic reviewer with a background in midwifery and clinical epidemiology and provided expert guidance in the design, implementation and write up of the paper.

\section{Funding}

LH was supported by the Stellenbosch University Collaborative Capacity Enhancement through Engagement with Districts (SUCCEED) project, funded by the USA Centers for Disease Control and Prevention (CDC) under GH151574, the South African University-based Technical Assistance aimed at Improving the quality of HIV/AIDS and related services in the Republic of South Africa under PEPFAR. Further support to complete this work was provided by the Global Minds PhD programme at KU Leuven (Grant: 3H180579). AR and IEW were supported by the Research, Evidence and Development Initiative (READ-It) project (project number 300342-104). READ-It is funded by aid from the UK government; however, the views expressed do not necessarily reflect the UK government's official policies.

\section{Availability of data and materials}

All data generated or analysed during this study are included in this published article and its supplementary information files. 


\section{Ethics approval and consent to participate}

Not applicable

\section{Consent for publication}

Not applicable

\section{Competing interests}

$\mathrm{LH}, \mathrm{AR}$, and IEW are authors on a qualitative systematic review on factors influencing linkage, adherence to ART and retention in care among PLWHIV in Sub-Saharan Africa, with IEW being the lead author. This review was not completed when the search was conducted and is included as an ongoing review. Authors note that their involvement in both projects was beneficial to understanding the existing evidence and were reflexive of any potential for biases in their conversations and dialogues that may have arose. The outcomes of either paper were not dependent on the other.

Received: 1 May 2020 Accepted: 6 January 2021 Published online: 11 February 2021

\section{References}

1. Sandelowski M, Barroso J, Voils Cl. Using qualitative metasummary to synthesize qualitative and quantitative descriptive findings. Res Nurs Health. 2007:30(1):99-111.

2. Ingrid E-W, Anke R, Lynne H, Sandy O, Paul G. Adherence, linkage and retention-in-care in antiretroviral treatment programmes in low and middle income countries: systematic review and synthesis of qualitative research. 2017;(PG-). Available from: http://www.crd.york.ac.uk/PROSPERO/display_ record.asp?ID=CRD42017057335 NS -

3. Noyes J, Booth A, Cargo M, Flemming K, Harden A, Harris J, Garside R, Hannes K, Pantoja T TJ. No Title. In: Cochrane Handbook for Systematic Reviews of Interventions version 61. 2020.

4. Aromataris E, Fernandez R, Godfrey C, Holly C, Khalil H, Tungpunkom P. Chapter 10: Umbrella Reviews. In: Aromataris E, Munn Z (Editors). JBI Manual for Evidence Synthesis. JBI, 2020. Available from https://synthesismanual.jbi. global. https://doi.org/10.46658/JBIMES-20-11.

5. Hunt H, Pollock A, Campbell P, Estcourt L, Brunton G. An introduction to overviews of reviews: planning a relevant research question and objective for an overview. Syst Rev [Internet]. 20181 [cited 2018 Sep 4];7(1):39. Available from: https://systematicreviewsjournal.biomedcentral.com/ articles/10.1186/s13643-018-0695-8

6. Pollock A. Cochrane overviews of reviews: exploring the methods and challenges. UK and Ireland Cochrane Symposium; 2016

7. May CR, Cummings A, Myall M, Harvey J, Pope C, Griffiths P, et al. Experiences of long-term life-limiting conditions among patients and carers: What can we learn from a meta-review of systematic reviews of qualitative studies of chronic heart failure, chronic obstructive pulmonary disease and chronic kidney disease? BMJ Open. 2016;6(10).

8. Toye F, Seers K, Hannink E, Barker K. A mega-ethnography of eleven qualitative evidence syntheses exploring the experience of living with chronic non-malignant pain. BMC Med Res Methodol [Internet]. 20171 [cited 2018 Aug 28];17(1):116. Available from: http://bmcmedresmethodol. biomedcentral.com/articles/10.1186/s12874-017-0392-7

9. Hannes K, Pearson A. Obstacles to the implementation of evidence-based practice in Belgium: a worked example of meta-aggregation. In: Synthesizing Qualitative Research [Internet]. Chichester, UK: John Wiley \& Sons, Ltd; 2012 [cited 2018 Aug 25]. p. 21-39. Available from: http://doi. wiley.com/10.1002/9781119959847.ch2

10. Bnurs KP, Cardiac G. Qualitative research synthesis: methodological 2015; 179-87.

11. Collaborators GBDCM, Bhutta ZA, Coates MM, Coggeshall M, Dandona L, Diallo K, et al. Global, regional, national, and selected subnational levels of stillbirths, neonatal, infant, and under-5 mortality, 1980-2015: a systematic analysis for the Global Burden of Disease Study 2015. Lancet [Internet]. 2016:(PG-). Available from: https://www.scopus.com/inward/record.uri?eid = 2-s2.0-84994071373\&doi $=$ https://doi.org/10.1016/S0140-6736(16)315756\&partnerlD $=40 \& \mathrm{md} 5=\mathrm{b} 82 \mathrm{~d} 5 \mathrm{~d} 41478 \mathrm{fee} 622 \mathrm{f6} 8081 \mathrm{c} 450 \mathrm{~b} 8254 \mathrm{NS}$ -

12. Global Health Observatory (GHO) data-HIV/AIDS [Internet]. WHO. World Health Organization; 2018 [cited 2018 Aug 27]. Available from: http://www. who.int/gho/hiv/en/

13. Joint United Nations Programme on HIV/AIDS (UNAIDS). 90-90-90: An ambitious treatment target to help end the AIDS epidemic [Internet]. 2014 [cited 2018 Aug 27]. Available from: http://www.unaids.org/sites/default/ files/media_asset/90-90-90_en_0.pdf

14. UNAIDS. 2017 Global HIV Statistics [Internet]. 2018 [cited 2018 Aug 27]. Available from: http://www.unaids.org/sites/default/files/media_asset/ UNAIDS_FactSheet_en.pdf

15. Wada N, Jacobson LP, Cohen M, French A, Phair J, Muñoz A. Practice of epidemiology cause-specific life expectancies after 35 years of age for human immunodeficiency syndrome-infected and human immunodeficiency syndrome-negative individuals followed simultaneously in long-term cohort studies, 1984-2008. 2013 [cited 2018 Aug 27]; Available from: http://aje.oxfordjournals.org/

16. Kobin $A B$, Sheth NU. Levels of adherence required for virologic suppression among newer antiretroviral medications. Ann Pharmacother [Internet]. 2011 [cited 2018 Aug 27];45(3):372-9. Available from: http://www.ncbi.nlm.nih. gov/pubmed/21386024

17. Eshaun-Williams I, Rohwer A, Hendricks L, Young T. Barriers and facilitators to linkage, adherence and retention among HIV positive patients: an overview of systematic reviews [Internet]. 2017 [cited 2018 Aug 27]. Available from: https://www.crd.york.ac.uk/prospero/display record. php?RecordID = 78155

18. Hannes K, Macaitis K. A move to more systematic and transparent approaches in qualitative evidence synthesis: update on a review of published papers. Qual Res. 2012;12(4):402-42.

19. Pollock M, Fernandes RM, Becker LA, Featherstone R, Hartling L. What guidance is available for researchers conducting overviews of reviews of healthcare interventions? A scoping review and qualitative metasummary. Syst Rev [Internet]. 201614 [cited 2018 Aug 19];5(1):190. Available from: http://systematicreviewsjournal.biomedcentral.com/articles/10.1186/s13643016-0367-5

20. Hannes K, Lockwood C. Pragmatism as the philosophical foundation for the Joanna Briggs meta-aggregative approach to qualitative evidence synthesis. J Adv Nurs [Internet]. 2011 Jul [cited 2018 Aug 25];67(7):1632-42. Available from: http://doi.wiley.com/10.1111/j.1365-2648.2011.05636.x

21. Hannes K, Petry $K$, Heyvaert M. The meta-aggregative approach to qualitative evidence synthesis : a worked example on experiences of pupils with special educational needs in inclusive education The meta-aggregative approach to qualitative evidence synthesis : a worked example on experie. 2017;(April 2018).

22. Lockwood C, Munn Z, Porritt K. Qualitative research synthesis: methodological guidance for systematic reviewers utilizing metaaggregation. Int J Evid Based Healthc. 2015;13(3):179-87.

23. Kaufman MR, Cornish F, Zimmerman RS, Johnson BT. Health behavior change models for HIV prevention and AIDS care: practical recommendations for a multi-level approach [Internet]. 2014 [cited 2018 Aug 27]. Available from: www.jaids.com

24. Hunt H, Pollock A, Campbell P, Estcourt L, Brunton G. An introduction to overviews of reviews: planning a relevant research question and objective for an overview. Syst Rev [Internet]. 2018 [cited 2018 Aug 28];7(1):39. Available from: http://www.ncbi.nlm.nih.gov/pubmed/29490699

25. Noyes J, Booth A, Flemming K, Garside R, Harden A, Lewin S, et al. Cochrane Qualitative and Implementation Methods Group guidance series-paper 3: methods for assessing methodological limitations, data extraction and synthesis, and confidence in synthesized qualitative findings. J Clin Epidemiol [Internet]. 2018 [cited 2018 Aug 28];97:49-58. Available from: http://www.ncbi.nlm.nih.gov/pubmed/29247700

26. Munthe-Kaas H, Bohren MA, Glenton C, Lewin S, Noyes J, Tunçalp Ö, et al. Applying GRADE-CERQual to qualitative evidence synthesis findings-paper 3: how to assess methodological limitations. Implement Sci [Internet]. 201825 [cited 2018 Aug 28];13(S1):9. Available from: https://implementationscience. biomedcentral.com/articles/10.1186/s13012-017-0690-9

27. Lockwood C, Munn Z, Porritt K. Qualitative research synthesis. Int J Evid Based Healthc [Internet]. 2015 Sep [cited 2018 Aug 25];13(3):179-87. Available from: http://content.wkhealth.com/linkback/openurl?sid = WKPTLP: landingpage\&an = 01787381-201509000-00010

28. Munn Z, Stern C, Aromataris E, Lockwood C, Jordan Z. What kind of systematic review should I conduct? A proposed typology and guidance for systematic reviewers in the medical and health sciences. BMC Med Res Methodol [Internet]. 2018 [cited 2018 Aug 27];18(1):5. Available from: http:// www.ncbi.nlm.nih.gov/pubmed/29316881

29. Carroll C, Booth A, Cooper K. A worked example of "best fit" framework synthesis: a systematic review of views concerning the taking of some 
potential chemopreventive agents. BMC Med Res Methodol [Internet]. 2011 [cited 2018 Aug 26];11:29. Available from: http://www.ncbi.nlm.nih.gov/ pubmed/21410933

30. Carroll C, Booth A, Leaviss J, Rick J. "Best fit" framework synthesis: refining the method. BMC Med Res Methodol [Internet]. 201313 [cited 2018 Aug 26];13(1):37. Available from: http://bmcmedresmethodol.biomedcentral.com/ articles/10.1186/1471-2288-13-37

31. Keller SC, Yehia BR, Eberhart MG, Brady KA. Accuracy of definitions for linkage to care in persons living with HIV HHS public access. J Acquir Immune Defic Syndr [Internet]. 2013 [cited 2018 27];63(5):622-30. Available from: https:// www.ncbi.nlm.nih.gov/pmc/articles/PMC3796149/pdf/nihms-483322.pdf

32. Wolfe D, Carrieri MP, Shepard D. Treatment and care for injecting drug users with HIV infection: a review of barriers and ways forward. Lancet (London, England) [Internet]. 2010 [cited 2018 Aug 27];376(9738):355-66. Available from: http://www.ncbi.nlm.nih.gov/pubmed/20650513

33. Ivo N. Azia, Ferdinand C Mukumbang, van Wyk B. Southern African journal of HIV medicine. [Internet]. Vol. 17, Southern African Journal of HIV Medicine. 2016 [cited 2018 Aug 27]. 8 p. Available from: https://sajhivmed. org.za/index.php/hivmed/article/view/476/875

34. Azia IN, Mukumbang FC, Van Wyk B. Barriers to adherence to antiretroviral treatment in a regional hospital in Vredenburg, Western Cape, South Africa. South Afr J HIV Med [Internet]. 201630 [cited 2018 Aug 27];17(1). Available from: https://sajhivmed.org.za/index.php/hivmed/article/view/476

35. Bucciardini R, Fragola V, Abegaz T, Lucattini S, Halifom A, Tadesse E, et al. Retention in care of adult HIV patients initiating antiretroviral therapy in Tigray, Ethiopia: a prospective observational cohort study. Okulicz JF, editor. PLoS One [Internet]. 20154 [cited 2018 Aug 27];10(9):e0136117. Available from: http://dx.plos.org/10.1371/journal.pone.0136117

36. Obai G, Mubeezi R, Makumbi F. Rate and associated factors of non-retention of mother-baby pairs in HIV care in the elimination of mother-to-child transmission programme, Gulu-Uganda: a cohort study. BMC Health Serv Res [Internet]. 201718 [cited 2018 Aug 27];17(1):48. Available from: http:// bmchealthservres.biomedcentral.com/articles/10.1186/s12913-017-1998-5

37. Young T, Rohwer A, Volmink J, Clarke M. What are the effects of teaching evidence-based health care (EBHC)? Overview of systematic reviews. PLoS One [Internet]. 2014 [cited 2018 Aug 25];9(1):e86706. Available from: http:// www.ncbi.n/m.nih.gov/pubmed/24489771

38. Innovation $\mathrm{VH}$. Covidence systematic review software [Internet]. Available from: https://support.covidence.org/help/how-can-i-citecovidence.

39. Methodology for JBI Umbrella Reviews [Internet]. 2014 [cited 2018 Sep 3]. Available from: www.joannabriggs.org

40. Tricco AC, Lillie E, Zarin W, O'Brien KK, Colquhoun H, Levac D, et al. PRISMA Extension for Scoping Reviews (PRISMA-SCR): Checklist and Explanation. Ann Intern Med [Internet]. 2018 Sep 4 [cited 2018 Sep 4]; Available from: http:// annals.org/article.aspx? doi = https://doi.org/10.7326/M18-0850

41. Ammon N, Mason S, Corkery JM. Factors impacting antiretroviral therapy adherence among human immunodeficiency virus-positive adolescents in Sub-Saharan Africa: a systematic review. Public Health [Internet]. 2018;157(0): 20-31. Available from: https://doi.org/10.1016/j.puhe.2017.12.010

42. Barroso J, Leblanc NM, Flores D. It's not just the pills: a qualitative metasynthesis of HIV antiretroviral adherence research. J Assoc Nurses AIDS Care [Internet]. 2017;28(4):462-78 Available from: https://doi.org/10.1016/j.jana. 2017.02.007.

43. Bolsewicz K, Debattista J, Vallely A, Whittaker A, Fitzgerald L. Factors associated with antiretroviral treatment uptake and adherence: a review. Perspectives from Australia, Canada, and the United Kingdom. AIDS Care Psychol Socio-Medical Asp AIDS/HIV. 2015;27(12):1429-38.

44. Bravo P, Edwards A, Rollnick S, Elwyn G. Tough decisions faced by people living with HIV: a literature review of psychosocial problems. Aids Rev [Internet]. 2010 [cited 2018 Sep 8];12(2):76-88. Available from: http://www. aidsreviews.com/resumen.php?id $=1087$ \&indice $=2010122 \& u=$ unp

45. Chop E, Duggaraju A, Malley A, Burke V, Caldas S, Yeh PT, et al. Food insecurity, sexual risk behavior, and adherence to antiretroviral therapy among women living with HIV: a systematic review. Health Care Women Int [Internet]. 2017;38(9):927-44. Available from: https://doi.org/10.1080/ 07399332.2017 .1337774

46. Colvin CJ, Konopka S, Chalker JC, Jonas E, Albertini J, Amzel A, et al. A systematic review of health system barriers and enablers for antiretroviral therapy (ART) for HIV-infected pregnant and postpartum women. PLoS One. $2014 ; 9(10)$.
47. Croome N, Ahluwalia M, Hughes LD, Abas M. Patient-reported barriers and facilitators to antiretroviral adherence in sub-Saharan Africa. Aids. 2017;31(7): 995-1007.

48. Engler $\mathrm{K}$, Lènàrt $\mathrm{A}$, Lessard $\mathrm{D}$, Toupin I, Lebouché B. Barriers to antiretroviral therapy adherence in developed countries: a qualitative synthesis to develop a conceptual framework for a new patient-reported outcome measure. AIDS Care - Psychol Socio-Medical Asp AIDS/HIV. 2018;30(May):17-28.

49. Ferguson L, Grant AD, Watson-Jones D, Kahawita T, Ong'ech JO, Ross DA. Linking women who test HIV-positive in pregnancy-related services to longterm HIV care and treatment services: a systematic review. Trop Med Int Heal. 2012;17(5):564-80.

50. Flores D, Leblanc N, Barroso J. Enrolling an retaining patients with human immunodeficiency virus (HIV) in their care: a metasynthesis of qualitative studies. Int J Nurs Stud. 2016;62(2):126-36.

51. Gaston GB, Alleyne-Green B. The impact of African Americans' beliefs about HIV medical care on treatment adherence: a systematic review and recommendations for interventions. AIDS Behav. 2013:17(1):31-40.

52. Geter A, Sutton MY, Hubbard McCree D. Social and structural determinants of HIV treatment and care among black women living with HIV infection: a systematic review: 2005-2016. AIDS Care [Internet]. 2018;30(4):409-16 Available from: https://www.tandfonline.com/doi/full/10.1080/09540121.201 8.1426827.

53. Govindasamy D, Ford N, Kranzer K. Risk factors, barriers and facilitators for linkage to antiretroviral therapy care: a systematic review. Aids. 2012;26(16): 2059-67.

54. Heestermans T, Browne JL, Aitken SC, Vervoort SC, Klipstein-Grobusch K. Determinants of adherence to antiretroviral therapy among HIV-positive adults in sub-Saharan Africa: a systematic review. BMJ Glob Heal [Internet] 2016;1 (4):e000125. Available from: http://gh.bmj.com/lookup/doi/10.1136/ bmjgh-2016-000125

55. Hodgson I, Plummer ML, Konopka SN, Colvin CJ, Jonas E, Albertini J, et al. A systematic review of individual and contextual factors affecting ART initiation, adherence, and retention for HIV-infected pregnant and postpartum women. PLoS One. 2014;9(11).

56. Katz IT, Ryu AE, Onuegbu AG, Psaros C, Weiser SD, Bangsberg DR, et al. Impact of HIV-related stigma on treatment adherence: systematic review and meta-synthesis. J Int AIDS Soc. 2013;16(3 Suppl 2).

57. Knettel BA, Cichowitz C, Ngocho JS, Knippler ET, Chumba LN, Mmbaga BT, et al. Retention in HIV care during pregnancy and the postpartum period in the option B+ era: a systematic review and meta-analysis of studies in Africa. JAIDS J Acquir Immune Defic Syndr [Internet]. 2017;77(PG-):1. Available from: http://insights.ovid.com/crossref?an = 00126334-900000000-96776

58. Lancaster KE, Cernigliaro D, Zulliger R, Fleming PF, Hill C, Carolina N, et al. Living with HIV in sub-Saharan Africa: a systematic review. African J AIDS Res. 2016;15(4):377-86.

59. Lankowski AJ, Siedner MJ, Bangsberg DR, Tsai AC. Impact of geographic and transportation-related barriers on HIV outcomes in sub-saharan Africa: a systematic review. AIDS Behav. 2014;18(7):1199-223.

60. Lazuardi E, Bell S, Newman CE. A "scoping review" of qualitative literature about engagement with HIV care in Indonesia. Sex Health. 2018;

61. Li H, Marley G, Ma W, Wei C, Lackey M, Ma Q, et al. The role of ARV associated adverse drug reactions in influencing adherence among HIVinfected individuals: a systematic review and qualitative meta-synthesis. AIDS Behav. 2017;21(2):341-51.

62. Lytvyn L, Siemieniuk RA, Dilmitis S, lon A, Chang Y, Bala MM, et al. Values and preferences of women living with HIV who are pregnant, postpartum or considering pregnancy on choice of antiretroviral therapy during pregnancy. BMJ Open. 2017;7(9):1-9.

63. Merten S, Kenter E, McKenzie O, Musheke M, Ntalasha H, Martin-Hilber A. Patientreported barriers and drivers of adherence to antiretrovirals in sub-Saharan Africa: a meta-ethnography. Trop Med Int Heal. 2010;15(SUPPL. 1):16-33.

64. Mey A, Plummer D, Dukie S, Rogers GD, O'Sullivan M, Domberelli A. Motivations and barriers to treatment uptake and adherence among people living with HIV in Australia: a mixed-methods systematic review. AIDS Behav [Internet]. 2016;(PG-1-34):1-34. Available from: https://www.scopus.com/ inward/record.uri?eid = 2-s2.0-84994388389\&partnerlD = 40\&md5 = fa7d78974dfe5881731fabdbc91d2be3 NS -

65. Mills EJ, Nachega JB, Bangsberg DR, Singh $S$, Rachlis B, Wu P, et al. Adherence to HAART: a systematic review of developed and developing nation patientreported barriers and facilitators. PLoS Med. 2006;3(11):2039-64. 
66. Morales-Aleman MM, Sutton MY. Hispanics/Latinos and the HIV continuum of care in the southern USA: a qualitative review of the literature, 2002-2013. AIDS Care - Psychol Socio-Medical Asp AIDS/HIV. 2014;26(12):1592-604.

67. Omonaiye $\mathrm{O}$, Kusljic $\mathrm{S}$, Nicholson $\mathrm{P}$, Manias E. Medication adherence in pregnant women with human immunodeficiency virus receiving antiretroviral therapy in sub-Saharan Africa: a systematic review. BMC Public Health [Internet]. 2018;18(805):1-20. Available from: https://doi.org/10.1186/ s12889-018-5651-y

68. Reisner SL, Mimiaga MJ, Skeer M, Safren SA. A review of HIV antiretroviral adherence and intervention studies among HIV-infected youth NIH public access [Internet]. Vol. 17, Top HIV Med. 2009 [cited 2018 Sep 8]. Available from: https://www.ncbi.nlm.nih.gov/pmc/articles/PMC3752381/pdf/nihms4 96536.pdf

69. Santer M, Ring N, Yardley L, Geraghty AWA, Wyke S. Treatment non-adherence in pediatric long-term medical conditions: systematic review and synthesis of qualitative studies of caregivers' views. BMC Pediatr. 2014;14(1):1-10.

70. Vervoort SCJM, Borleffs JCC, Hoepelman AIM, Grypdonck MHF. Adherence in antiretroviral therapy: a review of qualitative studies. AIDS. 2007;21 (May 2006):271-81.

71. Vitalis D. Factors affecting antiretroviral therapy adherence among HIVpositive pregnant and postpartum women: an adapted systematic review. Int J STD AIDS [Internet]. 2013;24(6):427-32. Available from: http://www. embase.com/search/results? subaction $=$ viewrecord\&from $=$ export\&id $=\mathrm{L3}$ 70229543\%5Cn , https://doi.org/10.1177/0956462412472807\%5Cn, http://sfx. library.uu.nl/utrecht?sid = EMBASE\&issn = 09564624\&id = doi:https://doi.org/ 10.1177/0956462412472807\&atitle $=$ Factors+affecting+antiret

72. Wasti SP, Van Teijlingen E, Simkhada P, Randall J, Baxter S, Kirkpatrick P, et al. Factors influencing adherence to antiretroviral treatment in Asian developing countries: a systematic review. Trop Med Int Heal. 2012;17(1):71-81.

73. Williams S, Renju J, Ghilardi L, Wringe A. Scaling a waterfall: a metaethnography of adolescent progression through the stages of HIV care in sub-Saharan Africa. J Int AIDS Soc [Internet]. 2017;20(1):1-17. Available from: https://doi.org/10.7448/IAS.20.1.21922

74. Moher D, Liberati A, Tetzlaff J, Altman DG, Group TP. Preferred Reporting Items for Systematic Reviews and Meta-Analyses: The PRISMA Statement. PLoS Med [Internet]. 200921 [cited 2018 Aug 27];6(7):e1000097. Available from: http://dx.plos.org/10.1371/journal.pmed.1000097

75. Mey A, Plummer D, Dukie S, Rogers GD, O'Sullivan M, Domberelli A. Motivations and barriers to treatment uptake and adherence among people living with HIV in Australia: a mixed-methods systematic review. AIDS Behav. 2017;21(2):352-85.

76. Booth A, Noyes J, Flemming K, Gerhardus A, Wahlster P, Van Der Wilt GJ, et al. Guidance on choosing qualitative evidence synthesis methods for use in health technology assessments of complex interventions 7 [Internet]. [cited 2018 Aug 28]. Available from: http://www.integrate-hta.eu/ downloads/

77. Majid, Umair; Vanstone M. Appraising qualitative research for evidence syntheses: a compendium of quality appraisal tools. 2018;

78. The Joanna Briggs Institute. Checklist for Systematic Reviews and Research Syntheses. Joanna Briggs Inst [Internet]. 2016; Available from: https:// joannabriggs.org/sites/default/files/2019-05/JBI_Critical_Appraisal-Checklist_ for_Systematic_Reviews2017_0.pdf

\section{Publisher's Note}

Springer Nature remains neutral with regard to jurisdictional claims in published maps and institutional affiliations.

Ready to submit your research? Choose BMC and benefit from:
- fast, convenient online submission
- thorough peer review by experienced researchers in your field
- rapid publication on acceptance
- support for research data, including large and complex data types
- gold Open Access which fosters wider collaboration and increased citations
- maximum visibility for your research: over 100M website views per year
At BMC, research is always in progress.
Learn more biomedcentral.com/submissions

